\title{
The evolution of widespread recombination suppression on the Dwarf Hamster (Phodopus) X chromosome
}

\author{
Emily C. Moore ${ }^{1,3}$, Gregg W. C. Thomas ${ }^{1}$, Sebastian Mortimer ${ }^{1}$, Emily E. K. Kopania ${ }^{1}$, Kelsie E. Hunnicutt ${ }^{2}$, Zachary J. \\ Clare-Salzler $^{1}$, Erica L. Larson ${ }^{2}$, and Jeffrey M. Good ${ }^{1,3}$ \\ ${ }^{1}$ Division of Biological Sciences, University of Montana, Missoula, MT, 59812; ${ }^{2}$ Department of Biological Sciences, University of Denver, Denver, CO, 80208 \\ This manuscript was compiled on January 4, 2022
}

The mammalian $X$ chromosome shows strong conservation among distantly related species, limiting insights into the distinct selective processes that have shaped sex chromosome evolution. We constructed a chromosome-scale de novo genome assembly for the Siberian dwarf hamster (Phodopus sungorus), a species reported to show extensive recombination suppression across an entire arm of the $\mathrm{X}$ chromosome. Combining a physical genome assembly based on shotgun and long-range proximity ligation sequencing with a dense genetic map, we detected widespread suppression of female recombination across $\sim 65 \%$ of the Phodopus $X$ chromosome. This region of suppressed recombination likely corresponds to the Xp arm, which has previously been shown to be highly heterochromatic. Using additional sequencing data from two closely-related species ( $P$. campbelli and $P$. roborovskii), we show that recombination suppression on $X p$ appears to be independent of major structural rearrangements. The suppressed Xp arm was enriched for several transposable element families and de-enriched for genes primarily expressed in the placenta, but otherwise showed similar gene densities, expression patterns, and rates of molecular evolution when compared to the recombinant $\mathrm{Xq}$ arm. Phodopus $\mathrm{Xp}$ gene content and order was also broadly conserved relative to the more distantly related rat $X$ chromosome. Collectively, these data suggest that widespread suppression of recombination has likely evolved through the transient induction of facultative heterochromatin on the Phodopus Xp arm without major changes in chromosome structure or genetic content. Thus, dramatic changes in the recombination landscape have so far had relatively subtle influences on overall patterns of $\mathrm{X}$-linked molecular evolution.

Sex chromosome evolution | sex-biased genes | transposable element accumulation | faster-X evolution |

The recurrent evolution of sex chromosomes represents one of the most extreme examples of genomic specialization. One hallmark of sex chromosome evolution is that the chromosome present only in the heterogametic sex often evolves a highly reduced, repetitive structure consisting primarily of a sex determination switch and genes involved in reproduction (reviewed in (1-3)). A common outcome of this process is an orthologous but heteromorphic sex chromosome pair with a single, degenerated sex-limited chromosome and a largely intact, recombining chromosome found in both sexes. Heteromorphic sex chromosomes are found in several of the most commonly studied organisms, including XY male heterogametic systems (most mammals and Drosophila), and ZW female heterogametic systems (birds and Lepidoptera). However, examination of these relatively ancient sex chromosomes reveals only the late stages of a presumably dynamic and complex process of structural evolution, functional specialization, and gene loss (1).
As sex chromosomes have been surveyed in more taxa, it has become clear that the amount of degeneration on the sexlimited chromosome (e.g., the Y or W) can be decoupled from chromosome age (4) and can vary between very closely related species (4-6). Recombination is the primary determinant of this dynamic process. Once a locus with sex-specific effects arises on a chromosome with a sex determination gene, sexually antagonistic selection should favor maintaining linkage between the two loci through suppressed recombination (2). As this process continues, regions with suppressed recombination are expected to lose functional genes and accumulate repetitive elements (7), and ultimately increase the proportion of the chromosome packaged as heterochromatin (8). Chromosomal inversions are thought to be a primary mechanism for recombination suppression, as they impede pairing and synapsis between homologous chromosomal regions in heterozygotes (9) and thereby link sexually-antagonistic alleles to sex determiners (10). Consistent with this model, complex structural rearrangements are a common feature differentiating heteromorphic sex chromosomes. However, inversions are only one possible mechanism for suppressed recombination (11), and it remains unclear if the rapid accumulation of inversions on sex chromosomes are a cause or consequence of broader recombination suppression (3).

In contrast, the $\mathrm{X}$ or $\mathrm{Z}$ chromosomes usually undergo free

\section{Significance Statement}

Sex chromosome evolution represents a dynamic process of genomic specialization that is thought to be dependent on evolution of recombination. Here we use genome sequencing and genetic mapping to show that one arm comprising the majority of the $X$ chromosome in a species of dwarf hamster has largely lost the ability to recombine in males and females. Although these dramatic shifts in recombination frequencies might eventually lead to sex chromosome degeneration, loss of recombination on this arm is associated with relatively minor changes in chromosome structure and gene contents in this species. These results underscore the conservation of the $\mathrm{X}$ chromosome across mammals, and allow us to test predictions about how genetic recombination influences sex chromosome evolution.

ECM, GWCT, and JMG conceived of the analyses, ECM, EEKK, KEEH, and ZJCS, and ELL collected tissues and constructed sequencing libraries, SM and ECM performed genome annotation, ECM and GWCT conducted all other analyses, and ECM, GWCT and JMG wrote the manuscript with feedback from all authors.

The authors declare no competing interests.

${ }^{3}$ To whom correspondence should be addressed. ECM, emily.christine.moore@gmail.com; JMG, jeffrey.good@mso.umt.eduu 
recombination in the homogametic sex (e.g., XX females or $\mathrm{ZZ}$ males). As a consequence, shared sex chromosomes often maintain some similarity to their inferred ancestral (autosomal) form and tend to be more conserved, but also have the potential to evolve rapidly. For example, mammalian X chromosomes tend to show broad conservation of gene content and order across placental mammals, reflecting a common origin and strong purifying selection related to dosage compensation $(12,13)$ and likely other functional dynamics $(11,14)$. At the same time, the $\mathrm{X}$ chromosome also shows several signatures of rapid evolution relative to the autosomes, including more rapid protein-coding evolution (i.e., faster-X evolution; reviewed in (15)) and enrichment for genes with both maleand female-specific functions $(16,17)$. Faster-X evolution is generally thought to reflect accelerated adaptive change due to immediate exposure of recessive beneficial mutations in males (18). However, both the strength of faster-X evolution and the pattern of sex-biased gene content varies considerably across taxa (1), likely reflecting variation in effective population sizes, the degree of female-biased transmission of the $\mathrm{X}$, mechanisms of dosage compensation (19), and epigenetic inactivation during certain developmental processes (e.g., meiotic sex chromosome inactivation in mammalian testis (20)). Understanding how all of these processes shape mammalian $\mathrm{X}$ chromosome evolution has been limited by the relatively ancient common origin and general structural conservation shared among taxa with sequenced genomes.

A rich history of mammalian cytogenetic research points to many potential exceptions to a conserved X paradigm that might help advance understanding of sex chromosome evolution $(21,22)$. One such exception may occur within the Cricetid rodent genus Phodopus, comprised of three species: Phodopus sungorus (Siberian or Djungarian hamster), it's closely-related sister taxon P. campbelli (Campbell's dwarf hamster), and P. roborovskii (Desert hamster). All three species are endemic to xeric steppe and desert habitats of central Asia (23-25). Phodopus sungorus and P. campelli have been developed as laboratory models to understand circadian and circannual plasticity in physiology, reproduction, and behavior (e.g., (26-28)). Phodopus species are also noteworthy for their unusually large sex chromosomes. The submetacentric $P$. sungorus $\mathrm{X}$ chromosome comprises $\sim 10 \%$ haploid female karyotype (29), approximately twofold more than the mammalian standard karyotype (30), with the karyotypically shorter (Xp) arm appearing largely as condensed constitutive (C-banding) heterochromatin $(29,31)$. The heterochromatic $\mathrm{Xp}$ arm is presumed to represent a massive expansion of highly repetitive (presumably non-genic) sequences in Phodopus (29), with a short distal pseudoautosomal region where all malespecific recombination occurs. Subsequent immunostaining (32) and genetic crossing (33) experiments have confirmed dramatic suppression of female recombination on the presumed $\mathrm{Xp}$ arm in $P$. sungorus, $P$. campbelli, and/or their F1 hybrids. However, the genic content of $\mathrm{Xp}$ is unknown and $\mathrm{X}$-linked incompatibilities are also primary determinants of hybrid male sterility (32) and disrupted hybrid placental development (33) in intercrosses between $P$. sungorus and $P$. campbelli, suggesting that functionally-relevant components of the $\mathrm{Xp}$ arm may be rapidly evolving.

Here we combine shotgun and long-range proximity ligation sequencing with genetic mapping data to construct the first

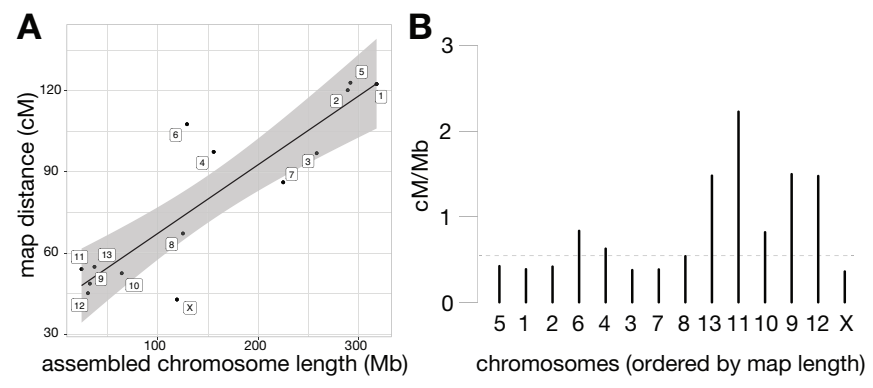

Fig. 1. Assembled length vs genetic map distance for all chromosomes. (A) Assembled chromosome length, determined by the total length of scaffolds anchored to the chromosome level, compared to the length of each chromosome in the genetic map. Black line shows the linear relationship between genetic and physical distance, with the gray shading indicating the $95 \%$ confidence interval of the linear model. (B) Recombination rate $(\mathrm{cM} / \mathrm{Mb})$ for all chromosomes, ordered by genetic length in the anchoring map. Genome-wide recombination rate is indicated with a dashed line. Chromosome numbers are retained from Brekke et al (2021), which named linkage groups based on genetic length from a de novo map.

chromosome-scale de novo genome assembly for the Siberian dwarf hamster ( $P$. sungorus). We then use multi-tissue transcriptomes, shotgun genome sequencing from $P$. campbelli and $P$. roborovskii, and comparative genome data from other mammals to examine the evolution of chromosome structure, genetic content, and protein-coding sequences across the Phodopus X chromosome.

\section{Results}

Phodopus chromosome-scale genome assembly and annotation. We generated an initial de novo assembly for a single $P$. sungorus female based on 742 million paired Illumina reads (150 bp PE, Illumina HiSeq X), resulting in near complete assembly of the estimated non-repetitive genome and $91 \%$ of the estimated total genome (estimated size 2,310 Megabases or $\mathrm{Mb}$ ). The total assembly covered $2,113.3 \mathrm{Mb}(98,297$ contigs, contig $\mathrm{N} 50=51.5$ kilobases or $\mathrm{kb}$ ) collected into 69,381 scaffolds (scaffold N50 = 79.7 kb). These data were then augmented with long-range proximity ligation data using complementary Dovetail Chicago and Hi-C methods. The end result was a highly contiguous scaffolded assembly (scaffold $\mathrm{N} 50=165.75 \mathrm{Mb}$; scaffold $\mathrm{N} 90=30.61 \mathrm{Mb}$ ) with $\sim 90 \%$ of the build contained within 14 scaffolds (Table S1); a dramatic improvement relative to a previously published draft Illumina shotgun assembly for this species (contig N50 = $2.2 \mathrm{~kb}$; scaffold $\mathrm{N} 50=4.8 \mathrm{~kb}(34)$ ). We then evaluated the quality of the genome assembly by re-mapping the short reads and comparing diploid genotype likelihoods for matched and mismatched bases relative to the haploid assembly of the longest scaffold for each chromosome as implemented with Referee (35). We estimated a per-base error rate of $4.22^{10-5}$, with $67.6 \%$ of evaluated bases receiving a quality score over 80 (Fig. S1).

We previously generated a genetic map for Phodopus (33) composed of 14 major linkage groups based on a backcross mapping experiment $[\mathrm{F} 1$ female $(P$. campbelli female $\mathrm{x} P$. sungorus male) $\mathrm{x} P$. campbelli male]. Both $P$. sungorus and $P$. campbelli have 13 mostly metacentric autosomes and a sex chromosome pair $(2 \mathrm{~N}=28 ; \mathrm{FN}=51(24,25))$, suggesting recovery of one linkage group per chromosome. To combine our genetic and physical maps, we mapped the ordered markers used to construct the genetic map to the genome assembly and 
anchored 37 scaffolds (99\% of the total genome assembly) into 14 chromosomal linkage groups. Nine of the large scaffolds corresponded to single linkage groups, likely representing eight of the 13 autosomes and the $\mathrm{X}$ chromosome. Four autosomes were recovered in two large scaffolds, while chromosome 5 was more fragmented with six assembled scaffolds (Table S2; numbering based on de novo genetic map lengths from (33).

Finally, we used available mouse protein data, Phodopus transcriptome data sampled from 10 tissues, and $a b$ initio predictions to generate and refine gene models. After filtering, we annotated 27,906 protein-coding and non-coding genes, including $87 \%$ (264 of 303) of the single-copy orthologs from the set of eukaryotic benchmarking genes (BUSCO, odb9; (36)). Of the annotated genes, 23,736 corresponded to proteincoding transcripts in the house mouse (mm10), of which 14,411 were 1:1 orthologs.

\section{Widespread suppression of X-linked recombination on the Xp} arm. Our previous mapping experiment indicated map compression on one end of the $\mathrm{X}$ chromosome (33). To quantify per chromosome recombination rates and the magnitude of $\mathrm{X}$-linked compression, we compared the number of base pairs anchored on each chromosome to its genetic length on the linkage map. Genome build size $(\mathrm{Mb})$ predicted $76.6 \%$ of the variance in recombination frequencies (map distance in centimorgans or $\mathrm{cM}$ ), with 11 chromosomes falling within the $95 \%$ confidence interval of the linear model (Fig.1A). The $\mathrm{X}$ chromosome was $\sim 1.7 \mathrm{x}$ the expected physical length predicted by genetic map distances (71.6 cM expected vs 41.3 cM observed; Fig.1). Recombination rates vary considerably across mammalian chromosomes and species, with X-linked rates typically lower in rodents in sex-averaged studies (37). We estimated an X-linked female recombination rate of 0.359 $\mathrm{cM} / \mathrm{Mb}$ (Fig.1B), compared to a genome-wide recombination rate of $0.55 \mathrm{cM} / \mathrm{Mb}$ which is similar to genome-wide rates in mice $(0.523 \mathrm{cM} / \mathrm{Mb})$ and rats $(0.551 \mathrm{cM} / \mathrm{Mb}$, rates from (37)).

Centromeres are dynamic chromosomal features that include diverse families of repeats (38) and can have functional epi-alleles that vary even within populations (39), making it difficult to localize centromeres in genome assemblies. Therefore, we examined crossover events across the assembled chromosomes and used the inherent reduction in recombination that results from condensed pericentric heterochromatin during meiosis to approximate the location of the centromeres in our assembly. Of the 13 autosomes, 10 displayed characteristic sinusoidal patterns with increased recombination near the distal regions of the chromosome arms (Fig. S2), as seen in other mammals with metacentric centromeres (40). Two chromosomes showed patterns consistent with an assembly error (chromosomes 9 and 11), which were localized by the bounds of the markers in the genetic map, but were unable to be further resolved without generating additional higher coverage long-read data for $P$. sungorus. In contrast, the $\mathrm{X}$ chromosome showed a partial sinusoidal pattern only for the first third ( $\sim 2 \mathrm{Mb})$ of the chromosome, with female recombination showing a reduction at the presumed centromere and then remaining suppressed along the rest of the $\mathrm{X}$ (Fig.2). Based on previous observations that recombination is mostly absent from the condensed Xp (32), we used the inflection point of recombination suppression to partition the $\mathrm{X}$ chromosome assembly into two arms, $\sim 42 \mathrm{Mb}(\mathrm{Xq})$ and $\sim 77 \mathrm{Mb}$ (Xp) long respectively. Under this model, the Xp arm (i.e., the
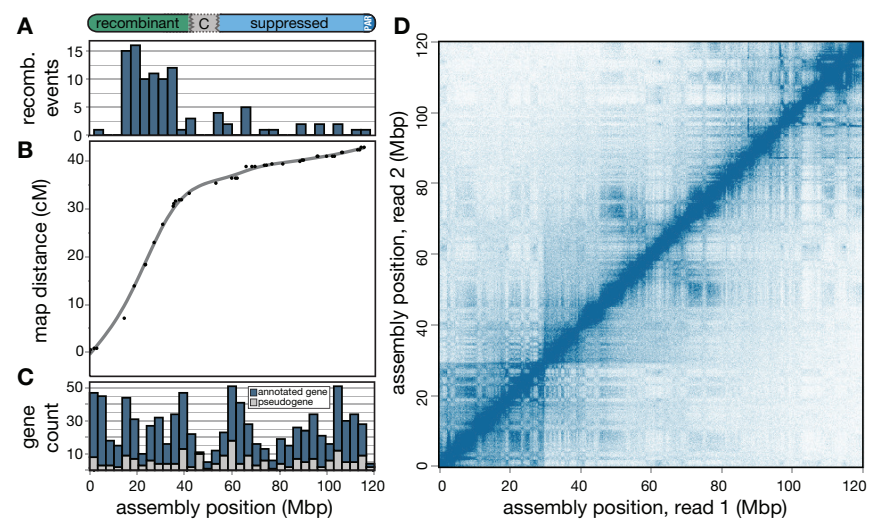

Fig. 2. Recombination suppression, gene content, and chromatin configuration of the Phodopus X chromosome. (A) Recombination events are predominantly localized to a single arm of the $X$ chromosome, which results in (B) an arm-specific map compression due to suppressed recombination. (C) Genes with complete gene models (blue) and putative pseudogenes with only expressed transcript support (gray) are distributed uniformly across the chromosome, despite the varying recombination levels. (D) $\mathrm{HiC}$ chromatin interaction plot, showing short- and long-range interactions between points on the $\mathrm{X}$ chromosome with a $250 \mathrm{Kbp}$ resolution and a square root coverage normalization.

shorter arm under standard convention) actually comprises $65 \%$ of the assembled X Phodopus chromosome (77 of 119 $\mathrm{Mb})$.

In the P. sungorus karyotype, the "shorter" Xp arm usually appears as condensed chromatin, which obscures its physical length relative to the less condensed Xq arm. The distal end of the Xp also harbors the pseudoautosomal region (PAR), which is the location of X-Y homology, synapsis, and all $\mathrm{X}$-linked recombination in males. To verify that the longer assembled arm was indeed the $\mathrm{Xp}$, we generated whole genome shotgun (WGS) sequence data for one outcrossed male $P$. sungorus and two reciprocal hybrids between $P$. sungorus and $P$. campbelli. We then used normalized patterns of female:male sequencing coverage and SNP density to localize the PAR to the distal $3 \mathrm{Mb}$ of the longer arm (Fig. S3). Hereafter, we refer to the suppressed and recombinant regions as the $\mathrm{Xp}$ and Xq chromosomal arms, respectively, with the caveat that additional work is needed to verify the exact location of the centromere. The overwhelming majority of observed female crossover events in our mapping panel occurred along the Xq arm, with an elevated estimated recombination rate of $0.78 \mathrm{cM} / \mathrm{Mb}$ relative to the Phodopus genome-wide rate of $0.55 \mathrm{cM} / \mathrm{Mb}$. Conversely, female recombination was severely suppressed across the entire Xp arm $(0.13 \mathrm{cM} / \mathrm{Mb})$, with $74 \mathrm{Mb}$ of $77 \mathrm{Mb}$ presumably experiencing little or no recombination in either sex.

The observation of dramatically reduced recombination across Xp is broadly consistent with heterochromatic suppression of recombination in females (32). However, our recombination data derives from $\mathrm{F} 1$ females between $P$. sungorus and P. campbelli and, therefore, could also reflect species-specific structural differences. To address this alternative hypothesis, we evaluated short read and long read mapping data, and compared chromatin configuration maps between the species. Comparisons of breakpoints identified by mapping of short read pairs support a $\sim 20 \mathrm{Mb}$ inversion on the distal region of Xp (spanning 95-115 Mp on the P. sungorus assembly, Fig. S4), which was further supported by 45 Nanopore long 

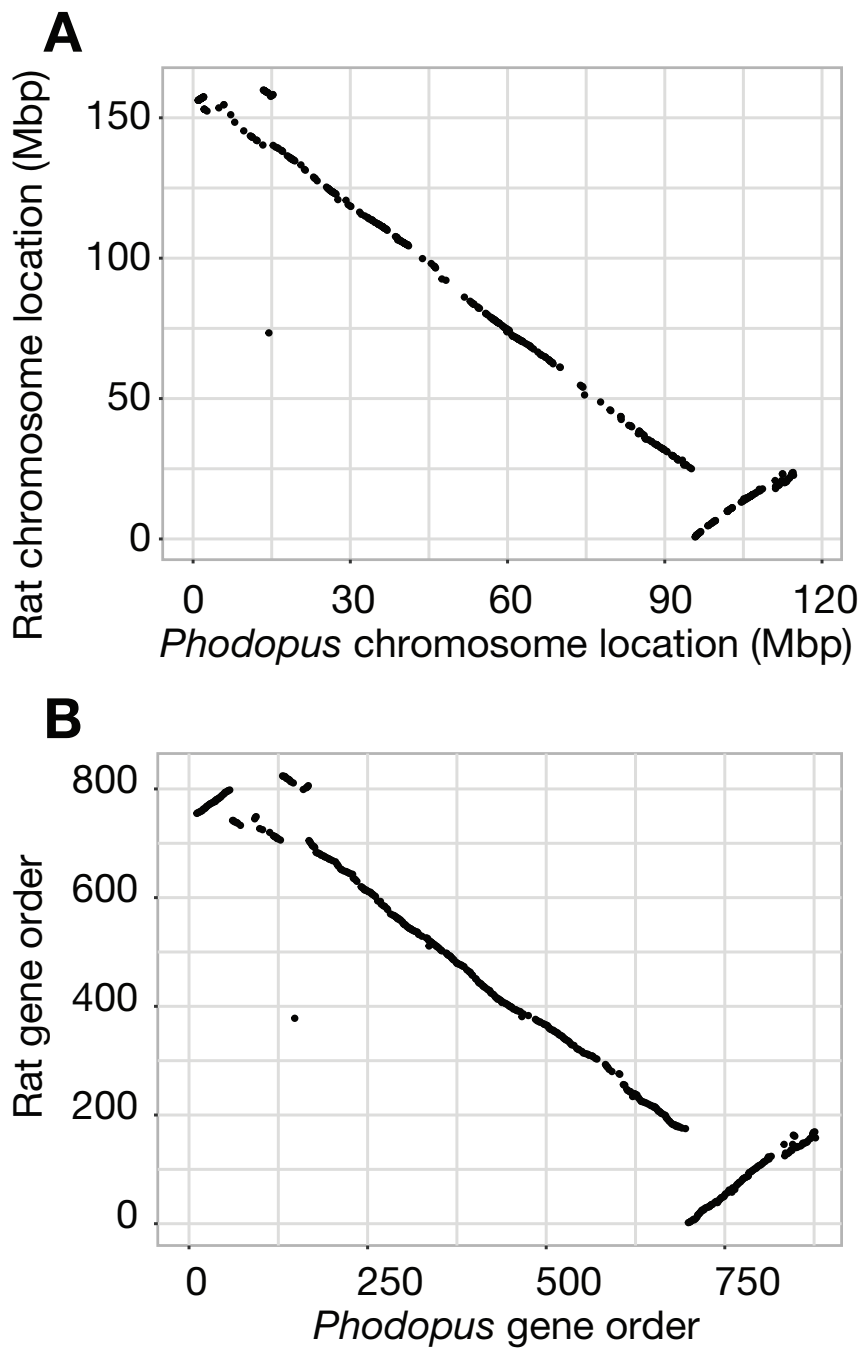

Fig. 3. Conservation of chromosome location and gene order between dwarf hamster and mouse. Gene start position $(\mathrm{Mb})(\mathrm{A})$ and relative order $(\mathrm{B})$ are largely conserved between dwarf hamster and rat along the $\mathrm{X}$ chromosome.

reads spanning the inferred breakpoints. Species differences in chromosome order were also detected from reductions in close chromosomal contacts in the $P$. campbelli $\mathrm{HiC}$ data at $115 \mathrm{Mb}$, compared to the P. sungorus assembly (Fig. S5C). This apparent inversion only encompassed $26 \%$ of the Xp arm (20 of 77 $\mathrm{Mb}$ ), and was insufficient to explain the broad recombination suppression extending well outside of this region.

Conservation of ancestral gene content and order despite the evolution of extensive repressive heterochromatin. Extraordinary conservation of gene orders is one of the hallmarks of mammalian X chromosome evolution (41), and may reflect strong selection preserving three-dimensional chromosomal structure of the X (14). To test whether dwarf hamsters have broadly maintained X chromosome synteny, we aligned the dwarf hamster $\mathrm{X}$ chromosome to the rat, mouse, dog, and human reference genomes. We also compared the relative order of our annotated genes to orthologous genes on the rat $\mathrm{X}$ chromosome. The distal end of the dwarf hamster Xp showed a similar pattern of rearrangement relative to both the dog and human references (Fig. S6). We detected several mouse- specific rearrangements across the $\mathrm{X}$ relative to other species, consistent with previous reports (Gibbs et al 2004; Brashear et al 2021). However, we found high conservation of X-linked gene orders between the $P$. sungorus dwarf hamster reference and rat, with an apparent inversion on the distal end of Xp and slight re-ordering of genes on the distal end of Xq (Fig.2). The inversion breakpoints on Xp match up with the inversion detected between Phodopus species (Fig. S4, S5), suggesting that $P$. campbelli retains the same, presumably ancestral, gene order as rat.

Constitutive heterochromatic chromosomal regions are generally assumed to be depauperate of expressed genes, reflecting the repressive effects of condensed chromatin on transcription and enrichment of tandem repeats near pericentromeric and telomeric chromatin (42). Given this and the relatively large size of the Phodopus X chromosome, the condensed and darkly staining Xp arm has been assumed to be highly repetitive and largely non-genic (29). Contrary to these predictions, we found that the Xp arm harbors approximately $54 \%$ of expressed genes on the Phodopus X chromosome (379 of 697 genes, Table S3), which is similar to the proportion of genes found on the orthologous and largely collinear arm in rat $57 \%$, 472 of 824 , Table S3). The Xp region did have fewer intact genes than expected given its proportional length in the dwarf hamster assembly (379 genes observed vs 432 genes expected, Fisher's exact $\mathrm{p}<0.0001)$. However, this pattern was also apparent in the orthologous region of the rat X (Fisher's exact $\mathrm{p}<0.0001)$, suggesting an ancestral reduction in gene density on $\mathrm{Xp}$ rather than gene loss subsequent to recombination suppression.

Even though we found many intact genes on the dwarf hamster $\mathrm{Xp}$, it is possible that the presence of heterochromatin could still impact gene function by broadly suppressing levels of gene expression. When we considered all the tissues except for testis (Fig. S7A), there was a slight reduction in expression on $\mathrm{Xq}$ relative to autosomes (pairwise Wilcoxon, $\mathrm{p}=0.018$ ) but no difference in the expression levels of $\mathrm{Xp}$ relative to the autosomes (pairwise Wilcoxon, $\mathrm{p}=0.702$ ) or $\mathrm{Xq}$ (pairwise Wilcoxon, $\mathrm{p}=0.126$ ). Testis was examined separately (Fig. S7B), as the X chromosome likely undergoes meiotic sex chromosome inactivation (MSCI) and postmeiotic repression during spermatogenesis, which is expected to reduce expression of many X-linked genes. Reductions in testis expression consistent with MSCI were apparent for both Xq (pairwise Wilcoxon, $\mathrm{p}<0.0001$ ) and $\mathrm{Xp}$ (pairwise Wilcoxon, $\mathrm{p}<0.0001$ ), with no difference in expression levels between the arms (pairwise Wilcoxon, $\mathrm{p}=0.44$ ).

When we included transcribed pseudogenes in the counts of gene distribution across arms, rat no longer showed a deficit of genes on the region orthologous to hamster Xp (660 of 1084; Fisher's exact $\mathrm{p}=0.1194)$, indicating more ancient loss of functional genes in this region in rodents that pre-dates recombination suppression in Phodopus. When we included putative pseudogenes in dwarf hamster (i.e., expressed genes in reference-guided transcriptomes without a complete gene model in the annotation), Phodopus continued to have slightly fewer genes than expected based on length (511 of 896; Fisher's exact $\mathrm{p}<0.0001)$. This may indicate that dwarf hamsters have lost pseudogene expression relative to the rat, though it is more likely that this difference merely reflects more complete rat annotation. 


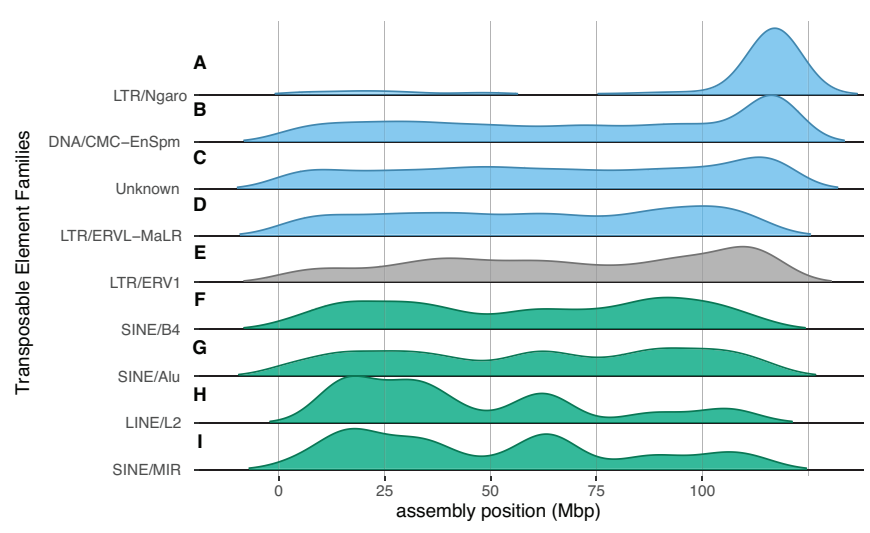

Fig. 4. Spatial distributions of the classes transposable elements significantly enriched on an arm of the Phodopus $X$ chromosome. Distributions of TE families across the Phodopus $\mathrm{X}$ assembly, position in Mbp. The top four classes (A-D; blue) are enriched on Xp only in dwarf hamster, ERV1 ( $E$, gray) is enriched on Xp in both dwarf hamster and rat, and the bottom four classes (F-I; green) are enriched on Xq in both dwarf hamster and rat. Significance determined by binomial test, see Table S4 for values.

Finally, if Xp were comprised of constitutive heterochromatin we should detect a clear signal of insulation from withinarm, long-range interactions from our $\mathrm{HiC}$ data as is seen in the inactive mouse $\mathrm{X}$ (43). In contrast, our $\mathrm{HiC}$ results from liver tissue of both $P$. sungorus (Fig.2D) and P. campbelli (Fig. $\mathrm{S} 5 \mathrm{~B})$ revealed smaller interspersed blocks of topographicallyassociated domains (TADs) that indicate active transcription (43). Thus, there was relatively little insulation on the arm with suppressed recombination, indicating that the heterochromatic state seen in metaphase karyotypes is tissue-dependent or perhaps transient in at least some transcriptionally active tissues.

Expansion of transposable element content on Xp. Another potential consequence of reduced recombination is the accumulation of transposable elements (TEs) due to a reduced efficacy of selection (44). To test for an accumulation of transposable elements and other repeats on $\mathrm{Xp}$, we annotated repetitive sequences in our genome build and the rat genome (version rn6; (45)). If suppressed recombination leads to the accumulation of TEs and repeats, we might expect to see a relative enrichment of repeat families on the dwarf hamster $\mathrm{Xp}$ relative to the homologous arm in rat. We found support for this hypothesis, as repeat families enriched on Xq were shared between the two species, but repeat families enriched on $\mathrm{Xp}$ were found enriched predominantly in hamster only. Dwarf hamster and rat shared patterns of TEs enriched on the female-recombining $\mathrm{Xq}$ and several transposable element families (e.g., SINEs and LINEs) were de-enriched on Xp and the homologous arm in rat (Fig.4F-I, Table S4), likely reflecting the shared evolutionary history of this region of the $\mathrm{X}$ chromosome. However, three of the four transposable element families enriched on Xp in dwarf hamster showed no evidence for repeat expansions in rat. Only the long terminal repeat (LTR) family ERV1 was also enriched in the orthologous region of the rat $\mathrm{X}$ (Fig.4E). Two families (DNA/EnSpm and LTR/MaLR, Fig.4B, D) enriched on Xp in dwarf hamster were significantly de-enriched in rat (Table S4). We also found that the Ngaro family of long terminal repeats (LTRs, Fig.4A) was highly enriched on only the recombination-reduced Xp (170 of 179 copies, Fisher's exact $\mathrm{p}<0.0001)$. In addition to TEs,
A

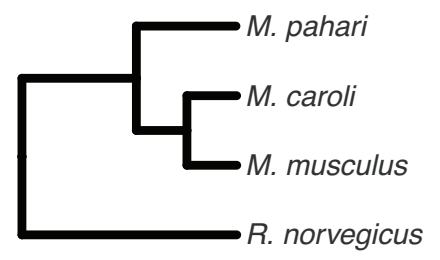

B
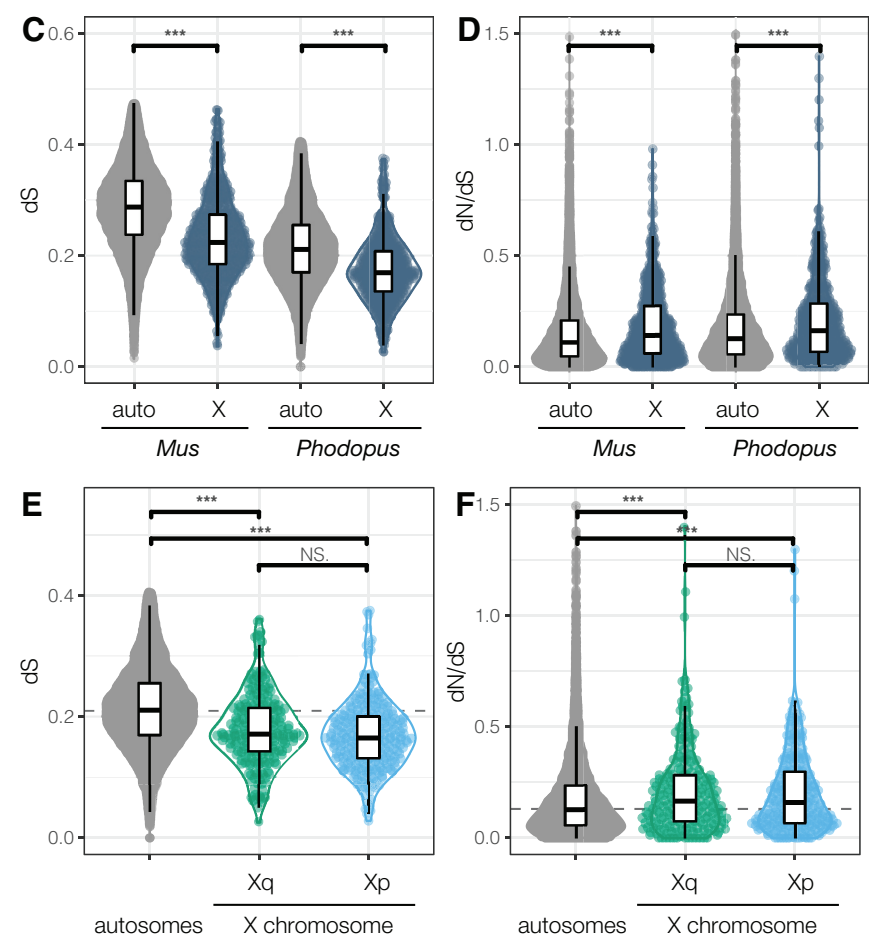

Fig. 5. Evolutionary rates on autosomes and $X$ chromosome arms in Phodopus Gene-specific rates of change in synonymous nucleotides (dS, C and E), and the ratio of non-synonymous to synonymous rates of change (dN/dS, D and F) for coding genes on the autosomes and $X$ chromosome arms, calculated separately for Mus (A) and Phodopus (B). For Phodopus, we also compared rates between the recombining and non-recombining arms of the $X$ chromosome $(E, F)$. Letters indicate significant differences within panel at $p<0.05$, Wilcoxon nonparametric multiple comparisons test. Dashed lines indicate genome-wide median.

we identified enrichment for other repetitive element classes (simple repeats, low complexity sequence, and repeat families of unknown identity, Fig.4C) on the Phodopus Xp, but not the recombining orthologous arm in rat. Thus, suppression of recombination appears associated with the accumulation of some repetitive sequence on the Phodopus X chromosome.

Molecular evolution of the $\mathbf{X}$ chromosome. To quantify patterns of molecular evolution on and off the $\mathrm{X}$ chromosome in dwarf hamsters, we evaluated the ratio of per site nonsynonymous $(\mathrm{dN})$ and synonymous $(\mathrm{dS})$ nucleotide changes in protein coding sequences $(\mathrm{dN} / \mathrm{dS})$. We estimated gene-wide rates in two parallel sets of four hamster or mouse species, each spanning similar evolutionary timescales (Fig.5A, B). Consistent with previous results $(46,47)$, we found faster-X protein-coding evolution for both mice $\left(\right.$ Mus $\mathrm{dN} / \mathrm{dS}_{\text {auto }}=0.15$; $\mathrm{dN} / \mathrm{dS}_{X}=0.19$, Wilcoxon $\left.\mathrm{p}<0.0001\right)$ and hamsters (Phodopus $\mathrm{dN} / \mathrm{dS}_{\text {auto }}=0.17 ; \mathrm{dN} / \mathrm{dS}_{X}=0.21 ;$ Fig.5D, Wilcoxon $\mathrm{p}<$ $0.0001)$. We also found a decrease in the rate of synonymous 
changes on the $\mathrm{X}$ chromosome for both groups (Wilcoxon Mus $\mathrm{p}<0.0001$, Phodopus $\mathrm{p}<0.0001$ ) without a corresponding reduction in non-synonymous changes (Fig.5C). Interestingly, dS was similar between the Xp and Xq arms (Fig.5E) and we did not observe differences in $\mathrm{dN} / \mathrm{dS}$ between the two arms (Fig.5F).

To test whether dwarf hamsters display sex-biased gene expression on the $\mathrm{X}$ chromosome, we examined gene expression across nine tissues (brain, heart, kidney, liver, muscle, spleen, uterus, placenta, and testis) using a specificity index $(\tau)$ to identify genes with tissue-enriched expression (Table S5; Fig.6). Approximately the same number of genes were expressed in each tissue (average $=17,904 \pm 542$ genes, range 15,11719,693 genes), and the majority of these genes were annotated as protein-coding based on orthology with Mus (93\%, 23,736 of 25,506 genes) The testis was enriched for tissue-specific expression; nearly one-third of all genes with a tissue specificity index $(\tau)$ greater than 0.8 were expressed in the testis $(2,918$ of 8,895 genes, Table S5), consistent with previous studies (48). We also detected enrichment on the $\mathrm{X}$ chromosome for testis-specific genes (164 genes, hypergeometric $\mathrm{p}<0.0001$, Fig.6A) and placenta-specific genes (50, hypergeometric $\mathrm{p}$ $<0.0001$, Fig.6B). When considered separately, each arm of the $\mathrm{X}$ chromosome was enriched for testis-specific expression (Xq, 67 genes, hypergeometric $\mathrm{p}=0.0005$; Xp, 97 genes, hypergeometric $\mathrm{p}<0.0001$, Fig.6A). However, only the femalerecombining $\mathrm{Xq}$ arm was enriched for placenta-specific genes (29 genes, hypergeometric $\mathrm{p}<0.0001$, Fig.6B).

When considering all genes expressed in the testis, rather than those with testis-specific expression, we no longer found an enrichment on the X chromosome (447 observed, 489 expected; Fisher's exact $\mathrm{p}=0.0572)$. The $\mathrm{X}$ showed a general de-enrichment of all placenta expressed genes ( 375 observed, 445 expected; Fisher's exact $\mathrm{p}=0.0037$ ), which was likely driven by a greater reduction of expressed genes on Xp (199 observed, 241 expected; Fisher's exact $\mathrm{p}=0.0048)$ compared to Xq (186 observed, 202 expected; Fisher's exact $p=0.1016$ ).

We also evaluated protein-coding sequence evolution in the four tissues with at least ten tissue-specific genes on each arm of the hamster X chromosome (brain, testis, placenta, and uterus, Fig. S8). In Mus, previous work has shown increased $\mathrm{dN} / \mathrm{dS}$ in male-biased genes on both the $\mathrm{X}$ and the autosomes, but no evidence for faster evolution in female-biased genes (46). In hamsters, we detected faster evolution of testis-biased genes across the autosomes and $\mathrm{X}$ compared to genome-wide values (Wilcoxon $\mathrm{p}<0.0001$ ), with $\mathrm{dN} / \mathrm{dS}$ for these genes on Xp slightly elevated relative to testis-biased autosomal genes $(\mathrm{p}=$ 0.037, Fig. S8B). We also found elevated dN/dS for X-linked placenta-specific genes, with placenta-biased genes evolving faster on Xq (Wilcoxon $p=0.012$, Fig. S8C). Tissue-specific genes are often associated with elevated $\mathrm{dN} / \mathrm{dS}$ relative to the genome-wide baselines (49), presumably reflecting reduced evolutionary constraints. In this admittedly limited survey, we detected significantly reduced rates for autosomal brain genes (Wilcoxon $\mathrm{p}<0.0001$ ), suggesting that tissue-specificity per se does not account for increased rates of X-linked evolution in dwarf hamsters.

\section{Discussion}

Mammalian X chromosomes are often characterized by specialized gene contents that also tend to be highly conserved
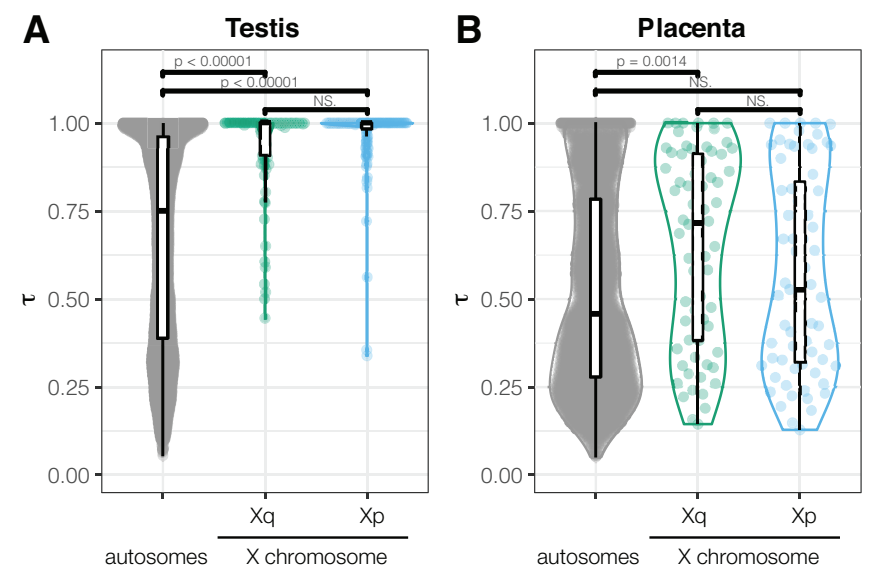

Fig. 6. Tissue specificity score $\tau$ for genes with highest expression in testis $(A)$ and placenta $(B)$, by $X$ chromosome region. Higher values of $\tau$ indicate higher tissue specificity in expression pattern.

between species, reflecting the late stages of a presumably dynamic process shaped by diverse selective pressures. Here we show that recombination suppression has evolved along most of an entire arm ( $65 \%)$ of the X chromosome in Phodopus dwarf hamsters. These findings provide an opportunity to evaluate the causes and evolutionary consequences of widespread recombination suppression on a mammalian sex chromosome.

The evolution of recombination suppression on the Phodopus $\mathbf{X}$ chromosome. Most studies that have examined recombination suppression in natural systems have focused on segregating structural inversions, which prevent the inverted region from forming successful Holliday junctions between alternate haplotypes. Pairing of sequence between sister chromatids is critical to successful crossing over during homologous recombination (50). On autosomes, segregating inversions have been found to prevent recombination between adaptive loci co-localized in the genome, linking together sets of genes for local adaptation (i.e., 'supergenes' in birds, butterflies, and Mimulus; reviewed in (51)). Inversions are also generally assumed to play a critical role in the evolution of heteromorphic sex chromosomes $(11,52)$, including stratified divergence between the mammalian $\mathrm{X}$ and $\mathrm{Y}$ chromosomes through a series of rearrangements (53). Indeed, inversions have been shown to maintain a heritable unit consisting of sexually-antagonistic alleles and a sex determination gene in young, homomorphic sex chromosomes in cichlid fishes (10). New inversions arise even on relatively old and conserved sex chromosomes - the mammalian $\mathrm{X}$ and avian $\mathrm{Z}$ chromosomes are enriched for inversions relative to autosomes (54). Theory predicts that the higher efficacy of selection on the $\mathrm{X}$ should limit the accumulation of deleterious mutations in inversions in the short term, which ultimately should facilitate higher inversion fixation rates in the long term when compared to autosomes (54).

Despite the intuitive link between structural changes and reduced recombination, we did not find large chromosomal rearrangements that easily explain the widespread suppression of recombination observed on the dwarf hamster Xp. Although our recombination map was generated with interspecies hybrids (33), the lack of support for an inversion model is unsurprising in this instance. The Xp usually appears to be highly condensed within Phodopus species, with clear evi- 
dence for suppression of female recombination on the presumed Xp arm within both $P$. sungorus and $P$. campbelli based on immunolocalization of the MLH1 mismatch repair protein (32). Thus, there are multiple lines of evidence for recombination suppression in crosses both with and between Phodopus species.

Karyotypes in $P$. sungorus and $P$. campbelli show strong C-banding of the Xp arm $(29,31)$, a hallmark of constitutive heterochromatin. However, these early studies also noted some variation between individuals in the condensation of $\mathrm{Xp}$ in different chromosome spreads, suggesting that the "constitutive" heterochromatin in this region was unlike the condensed, gene-poor regions of chromosomes found predominantly near centromeres and telomeres. Our findings confirm that the heterochromatic state of $\mathrm{Xp}$ is likely transient rather than classically constitutive. Phodopus Xp shows a chromatin state consistent with that of a transcriptionally active chromosome in liver tissue (Fig.2; Fig.S5), and the arm also has many genes expressed across tissues with no broad reduction in gene expression (Fig S7). One possible explanation is that $\mathrm{Xp}$ is condensed using canonical constitutive chromatin mechanisms, but in an inconsistent manner. Pericentromeric constitutive heterochromatin has been historically viewed as unchanging in the face of tissue type and environmental factors. However, research has increasingly uncovered dynamic complexity in the activity of the constitutive (H3K9me3) histone modification machinery (42), including expression of pericentromeric repeats during development and in actively proliferating tissues (55). The Phodopus X chromosome appears to have maintained broad conservation of gene order over the last $\sim 23$ million years since it shared a common ancestor with murine rodents (Fig.3, (56)). Therefore, many interspersed repeats associated with constitutive chromatin would presumably have had to have evolved along the Xp without major changes in synteny.

Perhaps more likely, condensation of Xp may be occurring via facultative heterochromatin pathways, such as that used in X chromosome inactivation (XCI) in females (57). These mechanisms are often context-dependent (e.g., sensitive to developmental timing and environmental cues) in addition to being sequence-dependent. XCI is initiated at the X inactivation center, where long noncoding RNA (lncRNA) Xist coats the $\mathrm{X}$ chromosome that will undergo inactivation (58). Xist then binds intermediary proteins to recruit the repressive Polycomb complex, a structure that mediates transcriptional silencing genome-wide (59). In this way, the universal machinery for the formation of facultative heterochromatin is recruited in an X-specific manner as XCI progresses. The X chromosome is prepared for heterochromatin formation even before Xist marks the inactive $\mathrm{X}$; the histone deacetylase HDAC3 is pre-loaded at enhancers along the $\mathrm{X}$ chromosome and plays a critical role in initiating chromosome condensation (57). Thus, the $\mathrm{X}$ chromosome has pre-existing sequences across its length that prime the formation of condensed heterochromatin in females. It is plausible such mechanisms could have been co-opted or expanded from the standard XCI pathway, inducing the transient heterochromatin state observed on Xp. Future studies examining histone marks indicative of facultative and constitutive heterochromatin are needed to test the epigenetic mechanisms underlying the formation of transient heterochromatin on $\mathrm{Xp}$.
Considerable literature has focused on the role that inversions play in generating recombination suppression (e.g., $(52,60,61))$. However, there remains relatively little direct evidence that rearrangements act as the primary proximate mechanism underlying the suppression of recombination on sex chromosomes (11). ). Regardless of the precise mechanisms, our results point to the relatively recent evolution of epigenetic suppression of recombination across a large portion of a mammalian X chromosome. It is also possible that other sequence changes, such as smaller inversions, nucleotide divergence (62), or TE accumulation (11), generally contribute to reduced X-linked recombination frequencies between $P$. sungorus and P. cambelli. However, such mechanisms cannot not explain suppression within these species (32). In other eukaryotic taxa, reports suggesting that non-inversion mechanisms may be at play in suppressed recombination have been more limited. Sylvioidea songbirds have a 'neo Z' region (i.e., an autosomal fusion on the $\mathrm{Z}$ chromosome) with a history of complex, progressive recombination suppression and repeat accumulation (63). Perhaps more relevant, recombination suppression in a group of Ascomycete fungi pre-dates inversions between species (64), with a proposed mechanism involving increased DNA methylation generated via the fungus-specific repeat-induced point (RIP) mutation repair pathway (65).

Evolutionary consequences of recombination suppression. In principle, a long-term reduction of recombination on Xp should lead to different evolutionary trajectories for the two arms of the Phodopus X, and provide insights into general models of sex chromosome differentiation. First, the accumulation of repetitive elements and structural variants (i.e., inversions, insertion-deletions (3)) are common features of sex chromosome evolution. The accumulation of sex-linked repetitive elements is generally thought to result from recombination suppression $(7,66)$ but see (11). Assuming our inference of a non-structural mechanism of suppression is correct, the enrichment for TEs on Xp between P. campbelli and P. sungorus is likely a consequence of a change in the recombination landscape. Some genomic sequences are predisposed to TE insertions (i.e., TE hotspots (67). However, we observed expansions of several TE families on the Phodopus Xp relative to the largely syntenic rat $\mathrm{X}$ chromosome, suggesting the differential accumulation of lineage-specific repeats independent of the ancestral sequence landscape of the rodent $\mathrm{X}$ chromosome.

The Phodopus system may also inform how recombination influences the dynamics of molecular evolution of the $\mathrm{X}$ chromosome. One outstanding question about the generation and maintenance of genetic diversity is whether recombination itself is a mutagenic process. This question has been difficult to answer because recombination rate is correlated with other features such as centromere location (40), gene density, and hotspot activity (68). In humans, sequencing evidence from sperm (69) and parent-offspring trios (70) demonstrates that recombination leads to mutation. However, it is less clear whether this phenomenon leads to sequence divergence across taxa (71). Our finding of similar levels of synonymous sequence divergence on the $\mathrm{Xp}$ and $\mathrm{Xq}$ arms suggests that changes in recombination frequencies have so far been a negligible contributor to substitution rates since the origin of $\mathrm{Xp}$ suppression in Phodopus.

The mammalian X chromosome generally shows lower synonymous substitution rates, presumably due to higher mu- 
tation rates in males (i.e., male-driven molecular evolution; (72) and female-biased transmission of the X chromosome (73). Despite a reduction in baseline mutation, theory predicts more efficient selection in males on the hemizygous X chromosome $(18,74)$, which may increase the fixation rate for beneficial alleles (i.e., the faster-X effect), especially in the testis and other male-specific tissues. Consistent with this, the $\mathrm{X}$ chromosomes of several mammal species show relatively higher rates of protein-coding evolution $(\mathrm{dN} / \mathrm{dS})$, which likely reflects more effective selection for recessive beneficial mutations (reviewed in (15)). Our finding of elevated X-linked $\mathrm{dN} / \mathrm{dS}$ in hamsters parallels results in mice and rats (Fig.5) and indicates that faster-X evolution is likely a general feature of rodent genomes. All else being equal, reduced recombination could limit the efficacy of natural selection on the Xp (i.e., Hill-Robertson interference (75)), which could lead to different rates of protein-coding evolution between Xp and Xq. We did not detect such differences, with both arms showing similarly elevated $\mathrm{dN} / \mathrm{dS}$ relative to the autosomes (Fig.5).

The $\mathrm{X}$ chromosome is also predicted to accumulate genes with sexually antagonistic variation (74), which can lead to an enrichment of sex-biased genes. Similar to predictions for faster-X evolution, the outcomes of selection for sex-biased gene contents are predicted to depend on the dominance of sexually antagonistic mutations. Male-biased genes are predicted to accumulate on the $\mathrm{X}$ if male-beneficial mutations are on average recessive. Likewise, given female-biased transmission, female-biased genes are predicted to accumulate on the $\mathrm{X}$ if female-beneficial mutations are on average dominant. Interestingly, these theoretical predictions play out differently in different systems (76). In Drosophila, the X appears to be strongly de-enriched for male-biased genes $(77,78)$, while mammals have been reported to be enriched for both maleand female-biased genes with some variation across studies $(20,79)$. In hamsters, we observed enrichment for both testisand placenta-specific genes, but not for other sex-specific tissues (i.e., uterus and ovary). These potentially conflicting patterns may be explained by inactivation of the paternal $\mathrm{X}$ chromosome in the placenta and other extraembryonic tissues of rodents $(80,81)$ resulting in predominantly maternal expression of X-linked placental genes. Thus, placental genes are functionally hemizygous, similar to X-linked genes in males. Notably, we found that testis-specific genes were enriched on both arms of the X, while enrichment of placental-specific genes was restricted to the female-recombining Xq arm. These patterns are based on small numbers of genes, but suggest that higher recombination may enhance the efficacy of sexually antagonistic selection in females.

Finally, these unusual evolutionary dynamics on the Phodopus X chromosome may also be influencing the rapid evolution of reproductive barriers between dwarf hamster species. Male hybrid sterility between female $P$. campbelli and male $P$. sungorus has been attributed to asynapsis of the sex chromosomes during meiosis (32), indicating that pairing may be inhibited by sequence or structural divergence that has accumulated on the PAR-containing Xp. Crosses between P. sungorus and $P$. campbelli) result in extreme hybrid placental overgrowth caused by a large-effect QTL near the P. sungorus Xq-Xp boundary (Fig. S9) that disrupts a network of autosomal genes expressed in the placenta (33). Understanding if and how the evolution of widespread recombination suppression is related to speciation awaits a more detailed dissection of the genetic and mechanistic bases of these hybrid incompatibilities.

\section{Materials and Methods}

Animal sampling. A single $P$. sungorus female was chosen for $d e$ novo genome sequencing and assembly from a breeding colony at the University of Montana (UM), originally established in 2011 descending from wild-caught animals collected 1981-1990 (82); see (83) for further details on P. sungorus and P. campbelli resources). The UM colony was maintained to maximize outbreeding, but levels of inbreeding are nonetheless elevated in these lines (Brekke et al. 2018). In addition to collecting liver from the reference individual for DNA sequencing, we collected seven snap-frozen tissues for use in transcriptome-based genome annotation (brain, heart, kidney, liver, muscle, spleen, and uterus). Testes from a single $P$. sungorus male from the same UM line were also collected to expand the annotation tissue panel. Augmenting the annotation panel, we collected and snap froze skin biopsies from molting $P$. sungorus females as part of an ongoing study on gene expression during seasonal molts. We sampled liver tissue from three additional males for shotgun genome sequencing: one outbred male $P$. sungorus was derived from a cross between a male from our original UM colonies and a female from supplemental stock kindly provided by Matthew Paul (University of Buffalo) in 2019, and two hybrid male embryos from reciprocal F1 crosses between $P$. sungorus and $P$. campbelli. Finally, we sequenced liver tissue from one female $P$. campbelli, derived from the UM research colony, and one male $P$. roborovskii sourced from the pet trade. All animal care and breeding was performed at UM with oversight of the University of Montana Institutional Animal Care and Use Committee (animal use protocols 039-13JGDBS-090413, 050-16JGDBS-082316 and 035-19JGDBS-062519).

Genome and transcriptome sequencing. Liver tissue was sent to Dovetail Genomics (Santa Cruz, CA) for generation of all sequencing data used in the de novo assembly, including Illumina whole genome shotgun sequencing (150 bp PE, Illumina HiSeq X) augmented with Dovetail's complementary Chicago (relying on artificial chromatin constructed in vitro) and Hi-C (relying on intact chromosomes in situ) long-range proximity ligation data. The libraries were prepared using Dovetail kits according to the manufacturer's protocol. For $\mathrm{Hi}-\mathrm{C}$ libraries, chromatin was fixed in place with formaldehyde in the nucleus and then extracted. Fixed chromatin was digested with DpnII, the 5' overhangs filled with biotinylated nucleotides, and free blunt ends ligated. After ligation, cross-linking was reversed and DNA was purified. Following purification, biotin not contained in the center of the ligated fragment was removed. Chicago libraries were prepared similarly, except DNA was removed from the nucleus and formed into artificial chromatin rather than fixed in situ. For all libraries, DNA was sheared to $\sim 350$ bp mean size and libraries were generated using Illumina-compatible adaptors.

We also generated an additional Hi-C library from $P$. campbelli liver tissue, processed following the same Dovetail protocols as the libraries generated for genome assembly. Whole genome sequencing libraries from $P$. campbelli, $P$. roborovskii, and three hybrid males were constructed using the NEBNext Ultra II DNA kit (New England Biolabs, E7645S). RNAseq libraries were constructed from all reference tissues at the University of Montana using an Illumina TruSeq Stranded mRNA library kit (Illumina, 20020595), following manufacturer's instructions, with the exception of the testis library, which was constructed at Novogene (Davis, CA). All tissues were sequenced on 3 lanes of 150 bp PE Illumina Hiseq 4000 (reference transcriptomes) and 3 lanes of $150 \mathrm{bp} \mathrm{PE} \mathrm{Illumina} \mathrm{Hiseq} \mathrm{X} \mathrm{(} \mathrm{HiC}$ and WGS libraries) at Novogene.

High molecular weight DNA for long read sequencing was extracted from the sequenced $P$. sungorus and $P$. campbelli females using the MagAttract HMW DNA kit (Qiagen, 67563) and assessed using a genomic DNA ScreenTape (Agilent 5067-5365) on a 2200 TapeStation (Agilent, G2965AA). Libraries were constructed and sequenced in the UM Genomics Core (Missoula, MT) using the MinION flowcell platform (Oxford Nanopore, sequencing kit SQK-LSK109 and flow cell FLO-MIN106D). 
Genome assembly and annotation. An initial shotgun sequencebased assembly was generated with 150 bp PE Illumina Hiseq data. Reads were trimmed with Trimmomatic (v 0.39, ILLUMINACLIP to remove adaptors, LEADING:20, SLIDINGWINDOW:13:20, MINLEN:23). The hybrid read-based/k-mer assembler Meraculous was used to generate a de novo assembly, with a constrained heterozygous (II) model with 79-mers and homozygous peak depth 40.0 selected for depth of coverage and balance between repetitive and heterozygous fractions (84). Reads from the additional Chicago and Hi-C libraries were used in conjunction with the de novo assembly as input for Dovetail's HiRise genome assembly pipeline (85). The first assembly iteration aligned Chicago library sequences to the draft assembly with a modified SNAP mapper (86). HiRise analyzed the distance between Chicago read pairs within scaffolds using a likelihood model, which was used to identify joins and misjoins for scaffolding. The second assembly iteration used the same strategy, scaffolding with the proximity-ligation $\mathrm{HiC}$ data.

To assess assembly quality on a per-site basis, we re-mapped the raw reads that went into the assembly and ran Referee (Thomas and Hahn, 2019) on the resulting BAM file to calculate genotype likelihoods while accounting for mapping quality. Sites in which the called base in the assembly does not match one of the two alleles in the most likely genotype are inferred as errors and can be corrected by inserting one of the alleles from that genotype.

We generated a reference-guided genetic map using data from our previously published Phodopus genetic map based on highthroughput genotypes (ddRAD tags) from 189 backcross individuals [F1 female ( $P$. campbelli female X. P. sungorus male) x P. campbelli male; (33)]. Processed RAD tags were aligned to the reference genome using BWA ( $\mathrm{Li}$ and Durban 2009), and variants were called using HaplotypeCaller (gatk, v4; (87)). The genetic map was constructed using rQTL as described previously (33), with the exception that duplicate markers were only dropped from the analysis if they were mapped to the same genomic scaffold. While these markers were redundant for identifying recombination events in the genetic map, they were informative for linking unanchored scaffolds and retained for scaffold placement.

We used the MAKER3 pipeline (v3.01.03; (88)) to annotate gene models using $a b$ initio and transcript-based evidence. Initial reference-guided de novo transcriptome assemblies were built with the Tuxedo pipeline (bowtie v2.3.4.3; (89) ; tophat v2.1.1 and cufflinks v2.2.1; (90)) including data from all reference tissues and published data previously used for de novo P. sungorus placental transcriptomes (80). These transcriptomes were given to the MAKER pipeline, which combined the Phodopus expression data, SNAP and Augustus ab initio gene prediction models, and protein homology to Mus to predict the location of genes and genic features. These genes were then matched with Mus (mm10) and Rattus (rnor6) identity through reciprocal best hits BLAST searches (91), using a max e-value cutoff of 1e-3 and then taking the longest remaining match between the two species.

Identification of the PAR and X-linked structural variation. For each all WGS samples, reads were trimmed (Trimmomatic v0.39; (92)) and aligned to the P. sungorus assembly using BWAmem (v0.7.17; (93)). Read depth was called using 'samtools depth' and averaged across $2500 \mathrm{bp}$ sliding windows (94). For the X chromosome, average coverage in each window was normalized by median coverage, and adjusted to reflect ploidy (ie. female ploidy is $2 \mathrm{~N}$ ). Next, we filtered out all windows that included bases overlapping with any annotated repeat (Repeatmasker (95)). We excluded regions annotated for STRs/low complexity sequences and all windows where the $P$. sungorus female had coverage over the $95 \%$ quantile of coverage (1.68X the median) in order to remove artifacts resulting from collapsed repeats or paralogous genes in the assembly. We used the same custom library to identify repetitive regions in both Phodopus and Rattus norvegicus (rnor6) as was used to repeat mask the genome for annotation.

We used four approaches to evaluate structural variation on the Phodopus X chromosome. First, we identified putative interspecific inversions using mapping discordance between $P$. campbelli and $P$. sungorus Illumina paired-end reads mapped to the $\mathrm{X}$ chromosome assembly (Breakdancer v1.3.6; (96)). We used analysis based on $P$. sungorus reads to control for any signal due to genome assembly error or repetitive content, and evaluated the difference between the reference and $P$. campbelli reads to find species-specific inversions by normalizing all counts the reference female, which was sequenced to higher sequence coverage. Second, we mapped Oxford Nanopore ultralong reads from $\mathrm{P}$. sungorus and $\mathrm{P}$. campbelli to the assembly using minimap2 (-ax map-ont; v2.17; (97)) and then used IGV (v2.7.2; (98)) to visually verify the large distal inversion by identifying that mapped across the inversion breakpoint, comparing these to a list of 45 Nanopore reads with regions mapped to either side of the inversion. Third, raw HiC reads from both $P$. sungorus and $P$. campbelli were processed with the Juicer pipeline to align and call $\mathrm{HiC}$ contact points (99), normalized using the Knight-Ruiz method. These matrices were then visualized and compared using Juicebox (v1.11.08 (100)). Fourth, we evaluated gene order/synteny across mammals. To assess synteny, we downloaded X chromosome assemblies from Ensembl (release 100) for Rattus norvegicus (rat, rnor6), Homo sapiens (human, $\mathrm{GrCh} 38$ ), and Canis familiaris (domestic dog, CanFam3.1). For each species, we used minimap2 (97) to align conserved segments from the $P$. sungorus $\mathrm{X}$ chromosome to the target reference (-asm20). Gene order was also evaluated by comparing the locations of Phodopus genes with those of the rat genes identified through reciprocal BLAST (described above). Recombination rate was evaluated using the RAD markers used to anchor the genome to the genetic map, using only the sequence length between markers to avoid including sequence without sampled recombination data.

Gene expression. We performed gene expression analysis using Hisat2 (101) to map reads to the genome assembly, and then used the MAKER-generated annotation to count reads with FeatureCounts (102). We allowed for multiple mapping but proportionally distributed multiply mapped reads across target genes. Counts were normalized using transcripts per million (TPM), and $\log 2$ transformed for analysis. All placenta samples were quantified following reference alignment, and the average TPM across samples was used to evaluate tissue specificity. We calculated the tissue specificity metric $\tau$ as described, using the established cutoff of $\tau 0.8$ (48) Expressed genes were also evaluated for overlap protein-coding molecular evolution metrics (see below).

Molecular evolution. We downloaded orthologous protein coding sequences for five species from Ensembl (release 100): $R$. norvegicus, M. musculus, M. caroli, M. pahari, and C. griseus. We used these sequences in conjunction with the $P$. sungorus genome and reference-guided assemblies of $P$. campbelli and $P$. roborovskii, constructed using iterative mapping to the $P$. sungorus to reduce reference bias. These iterative assemblies were as implemented with a modified version of pseudo-it (v.beta $3.1 ;(103)$ ) that incorporates insertion-deletion variation. For each species, we ran pseudo-it for four iterations and used the UCSC liftOver tool (University of California, Santa Cruz Genomics Institute) to transfer P. sungorus gene annotations to each pseudo-assembly.

We split the eight species into two groups of four species that spanned approximate similar evolutionary timescales. For the hamster group (Cricitidae: P. sungorus, P. campbelli, P. roborovskii, and C. griseus), we used BLAST (91) to obtain orthologous protein coding transcripts between the $\mathrm{C}$. griseus genome and $P$. sungorus by setting a max e-value cutoff of 1e-3 and then taking the longest remaining match between the two species. This resulted in 16,569 single-copy orthologs between these five species. For the mouse/rat group (Muridae: M. musculus, M. caroli, M. pahari, and R. norvegicus), we used Ensembl annotation to identify 15,772 single-copy orthologs. We aligned orthologous coding-sequences in both groups using MACSE (104), which produces in-frame codon alignments while accounting for frameshifts. First, sequences that contain non-homologous sequences were trimmed with the trimNonHomologousFragments sub-program. In some cases, this removed an entire sequence within the orthogroup, and this entire group was removed from subsequent steps. In total, 2,498 genes were removed from the hamster group and 266 were removed from the mouse group. Next, we aligned sequences with the main MACSE program, alignSequences (104). We followed this with a post-alignment trimming step using the trimAlignment sub-program, after which we calculated summary statistics for each alignment and removed those that were short $(<100 \mathrm{bp})$, contained a high proportion of sites with gaps or missing data $(>20 \%)$, contained identical sequences, or contained premature stop codons. In total, using these filters we removed 527 
genes from the hamsters and 79 genes from the mice. Following all alignment, trimming and filtering steps, we recovered 13,546 in-frame codon alignments for the hamsters and 15,429 from the mice.

Using these alignments, for both groups, we estimated maximumlikelihood gene trees using IQ-Tree (105) and estimated dN and dS for each gene using the corresponding gene trees in order to minimize the potential effects of discordance (106). Species level phylogenies were also verified from maximum likelihood analysis of a concatenated alignment (Fig.5A, B), but were not used for the analysis of molecular evolution. We used HyPhy (107) to estimate dN/dS using the FitMG94.bf model (https://github.com/veg/hyphy-analyses), estimating a single $\mathrm{dN} / \mathrm{dS}$ value per gene and summarizing $\mathrm{dN}$ and dS separately by summing their values over all branches (Fig.55). For each dataset, we flagged genes with dS values above the top 98th percentile of all genes as having potentially poor alignments and filtered them from subsequent analysis (Fig. S10; 271 Cricitidae genes and 309 Muridae genes).

Statistical analysis. All basic statistical tests were performed in $\mathrm{R}$ v 4.1.0 (108). Non-parametric models were performed with the Kruskal Wallace test function (kruskal.test) and posthoc pvalues were evaluated with pairwise.wilcox.test. Linear models we performed using $\mathrm{lm}$ function for standard models, and lmer for mixed models. Enrichment was tested either with hypergeometric test (for enrichment of categories, phyper) or Fisher's exact binomial test (for enrichment based on expected distributions from length, binom.test). Multiple testing was corrected for used the Bonferroni Holm method.

ACKNOWLEDGMENTS. We thank the Good lab for helpful comments on data analysis and interpretation. Ned Place, Matthew Paul, and Robert Johnston provided animal stocks. Kelly Carrick, Jess Wexler, and the University of Montana LAR staff assisted with animal care. Mark Daly, Thomas Swale, and Dovetail Genomics LLC performed genome sequencing and assembly. Denghui David Xing performed Nanopore sequencing at the UM Genomics Core. This research was supported by grants from the Eunice Kennedy Shriver National Institute of Child Health and Human Development of the National Institutes of Health (R01-HD094787 to JMG), a Matching Funds Award from Dovetail Genomics, and the National Science Foundation Graduate Research Fellowship Program (EEKK: DGE-1313190, and KEH DGE-2034612). This study included research conducted in the University of Montana Genomics Core, supported by a grant from the M. J. Murdock Charitable Trust (to JMG). Computational resources and support from the University of Montana's Griz Shared Computing Cluster (GSCC), supported by grants from the Nation Science Foundation (CC-2018112 and OAC-1925267, JMG co-PI), contributed to this research.

\section{References.}

1. Bachtrog D, et al. (2014) Sex determination: Why so many ways of doing it? PLOS Biology 12(7):e1001899.

2. Charlesworth $D$ (2021) When and how do sex-linked regions become sex chromosomes? Evolution 75(3):569-581.

3. Wright AE, Dean R, Zimmer F, Mank JE (2016) How to make a sex chromosome. Nat Commun 7:12087.

4. Darolti I, et al. (2019) Extreme heterogeneity in sex chromosome differentiation and dosage compensation in livebearers. Proceedings of the National Academy of Sciences 116(38):19031-19036

5. Bracewell RR, Bentz BJ, Sullivan BT, Good JM (2017) Rapid neo-sex chromosome evolution and incipient speciation in a major forest pest. Nature Communications 8(1):1593.

6. Peichel CL, et al. (2020) Assembly of the threespine stickleback y chromosome reveals convergent signatures of sex chromosome evolution. Genome Biology 21(1):177.

7. Charlesworth B, Langley C, Stephan W (1986) The evolution of restricted recombination and the accumulation of repeated dna sequences. Genetics 112(4):947-962.

8. Zhou Q, et al. (2013) The epigenome of evolving drosophila neo-sex chromosomes: Dosage compensation and heterochromatin formation. PLOS Biology 11(11):e1001711.

9. Kirkpatrick M (2010) How and why chromosome inversions evolve. PLOS Biology 8(9):e1000501.

10. Roberts RB, Ser JR, Kocher TD (2009) Sexual conflict resolved by invasion of a novel sex determiner in lake malawi cichlid fishes. Science 326(5955):998-1001.

11. Furman BLS, et al. (2020) Sex chromosome evolution: So many exceptions to the rules. Genome Biology and Evolution 12(6):750-763.

12. Ohno S (1967) Sex Chromosomes and Sex Linked Genes. (Springer Verlag, Berlin).
13. Pessia E, Makino T, Bailly-Bechet M, McLysaght A, Marais GAB (2012) Mammalian $x$ chromosome inactivation evolved as a dosage-compensation mechanism for dosage-sensitive genes on the $x$ chromosome. Proceedings of the National Academy of Sciences 109(14):53465351

14. Brashear WA, Bredemeyer KR, Murphy WJ (2021) Genomic architecture constrained placental mammal x chromosome evolution. Genome Res 31(8):1353-1365.

15. Meisel RP, Connallon $T$ (2013) The faster-x effect: integrating theory and data. Trends in genetics : TIG 29(9):537-544.

16. Reinius B, et al. (2012) Abundance of female-biased and paucity of male-biased somatically expressed genes on the mouse x-chromosome. BMC Genomics 13(1):607.

17. Wang X, et al. (2008) Transcriptome-wide identification of novel imprinted genes in neonatal mouse brain. PLoS One 3(12):e3839.

18. Charlesworth B, Coyne JA, Barton NH (1987) The relative rates of evolution of sex chromosomes and autosomes. The American Naturalist 130(1):113-146.

19. Mank JE, Vicoso B, Berlin S, Charlesworth B (2010) Effective population size and the faster-x effect: Empirical results and their interpretation. Evolution 64(3):663-674.

20. Khil PP, Smirnova NA, Romanienko PJ, Camerini-Otero RD (2004) The mouse $x$ chromosome is enriched for sex-biased genes not subject to selection by meiotic sex chromosome inactivation. Nature Genetics 36(6):642-646.

21. Couger MB, et al. (2021) Sex chromosome transformation and the origin of a male-specific $x$ chromosome in the creeping vole. Science 372(6542):592-600.

22. Veyrunes F, et al. (2008) Bird-like sex chromosomes of platypus imply recent origin of mammal sex chromosomes. Genome Research 18(6):965-973.

23. Ross PD (1994) Phodopus roborovskii. Mammalian Species 459:1-4.

24. Ross PD (1995) Phodopus campbelli. Mammalian Species 503:1-7.

25. Ross PD (1998) Phodopus sungorus. Mammalian Species 595:1-9.

26. Steinlechner S, Heldmaier G, Becker H (1983) The seasonal cycle of body weight in the djungarian hamster: Photoperiodic control and the influence of starvation and melatonin. Oecologia 60(3):401-405.

27. Surbhi, Schatz KC, Kyne RF, Nelson RJ, Paul MJ (2019) Photoperiod regulates hypothalamic mir-155 gene expression in female, but not male, siberian hamsters (phodopus sungorus) Behav Neurosci 133(2):240-246.

28. Wynne-Edwards K (1995) Biparental care in djungarian but not siberian dwarf hamsters (phodopus). Animal Behaviour 50:1571-1585.

29. Haaf T, Weis H, Schmid M (1987) A comparative cytogenetic study on the mitotic and meiotic chromosomes in hamster species of the genus phodopus (rodentia, cricetinae). Zeitschrift für Säugetierkunde 52:281-290.

30. Pathak S, Stock AD (1974) The $x$ chromosomes of mammals: Karyological homology as revealed by banding techniques. Genetics 78(2):703-714.

31. Gamperl R, Vistorin G, Rosenkranz W (1977) New observations on the karyotype of the djungarian hamster, phodopus sungorus. Experientia 15(8):1020-1021.

32. Bikchurina $\mathrm{TI}$, et al. (2018) Chromosome synapsis and recombination in male-sterile and female-fertile interspecies hybrids of the dwarf hamsters (phodopus, cricetidae). Genes (Basel) $9(5)$.

33. Brekke TD, et al. (2021) X chromosome-dependent disruption of placental regulatory networks in hybrid dwarf hamsters. Genetics 218(1).

34. Bao R, et al. (2019) Genome sequencing and transcriptome analyses of the siberian hamster hypothalamus identify mechanisms for seasonal energy balance. Proceedings of the National Academy of Sciences 116(26):13116-13121.

35. Thomas GWC, Hahn MW (2019) Referee: Reference assembly quality scores. Genome Biology and Evolution 11(5):1483-1486.

36. Simao FA, Waterhouse RM, loannidis P, Kriventseva EV, Zdobnov EM (2015) Busco: assessing genome assembly and annotation completeness with single-copy orthologs. Bioinformatics 31(19):3210-2.

37. Dumont BL, Payseur BA (2008) Evolution of the genomic rate of recombination in mammals. Evolution 62(2):276-294

38. Hartley G, O'Neill RJ (2019) Centromere repeats: Hidden gems of the genome. Genes (Basel) 10(3).

39. Purgato S, et al. (2015) Centromere sliding on a mammalian chromosome. Chromosoma 124(2):277-87.

40. Jensen-Seaman MI, et al. (2004) Comparative recombination rates in the rat, mouse, and human genomes. Genome Res 14(4):528-38.

41. Ohno S (1973) Ancient linkage groups and frozen accidents. Nature 244(5414):259-262.

42. Saksouk N, Simboeck E, Dejardin J (2015) Constitutive heterochromatin formation and transcription in mammals. Epigenetics and Chromatin 8(3).

43. Giorgetti L, et al. (2016) Structural organization of the inactive $x$ chromosome in the mouse Nature 535(7613):575-579.

44. Dolgin ES, Charlesworth B (2008) The effects of recombination rate on the distribution and abundance of transposable elements. Genetics 178(4):2169-77.

45. Gibbs RA, et al. (2004) Genome sequence of the brown norway rat yields insights into mammalian evolution. Nature 428(6982):493-521.

46. Kousathanas A, Halligan DL, Keightley PD (2014) Faster-x adaptive protein evolution in house mice. Genetics 196(4):1131-+.

47. Larson EL, Keeble S, Vanderpool D, Dean MD, Good JM (2016) The composite regulatory basis of the large x-effect in mouse speciation. Molecular Biology and Evolution 34(2):282295.

48. Kryuchkova-Mostacci N, Robinson-Rechavi M (2016) A benchmark of gene expression tissue-specificity metrics. Briefings in Bioinformatics 18(2):205-214.

49. Winter EE, Goodstadt L, Ponting CP (2004) Elevated rates of protein secretion, evolution, and disease among tissue-specific genes. Genome Research 14(1):54-61.

50. Hunter N (2015) Meiotic recombination: The essence of heredity. Cold Spring Harb Perspect Biol 7(12).

51. Thompson MJ, Jiggins CD (2014) Supergenes and their role in evolution. Heredity 113(1):1- 
bioRxiv preprint doi: https://doi.org/10.1101/2021.11.15.468705; this version posted January 4, 2022. The copyright holder for this preprint (which was not certified by peer review) is the author/funder. All rights reserved. No reuse allowed without permission.

52. Olito C, Abbott JK (2020) The evolution of suppressed recombination between sex chromosomes by chromosomal inversions. bioRxiv p. 2020.03.23.003558.

53. Lahn BT, Page DC (1999) Four evolutionary strata on the human $x$ chromosome. Science 286(5441):964-7.

54. Connallon T, et al. (2018) Local adaptation and the evolution of inversions on sex chromosomes and autosomes. Philosophical Transactions of the Royal Society B: Biological Sciences 373(1757):20170423.

55. Rudert F, Bronner S, Garnier JM, Dolle P (1995) Transcripts from opposite strands of gamma satellite dna are differentially expressed during mouse development. Mammalian Genome 6:76-83.

56. Schenk JJ, Rowe KC, Steppan SJ (2013) Ecological opportunity and incumbency in the diversification of repeated continental colonizations by muroid rodents. Syst Biol 62(6):83764.

57. Zylicz JJ, Heard E (2020) Molecular mechanisms of facultative heterochromatin formation: An x-chromosome perspective. Annu Rev Biochem 89:255-282.

58. Penny GD, Kay GF, Sheardown SA, Rastan S, Brockdorff N (1996) Requirement for xist in $x$ chromosome inactivation. Nature 379(6561):131-137.

59. Pintacuda G, et al. (2017) hnrnpk recruits pcgf3/5-prc1 to the xist rna b-repeat to establish polycomb-mediated chromosomal silencing. Molecular Cell 68(5):955-969.e10.

60. Charlesworth $D(2017)$ Evolution of recombination rates between sex chromosomes. Philos Trans R Soc Lond B Biol Sci 372(1736).

61. Fuller ZL, et al. (2020) Extensive recombination suppression and epistatic selection causes chromosome-wide differentiation of a selfish sex chromosome in drosophila pseudoobscura. Genetics 216(1):205-226.

62. Lukacsovich T, Waldman AS (1999) Suppression of intrachromosomal gene conversion in mammalian cells by small degrees of sequence divergence. Genetics 151(4):1559-1568.

63. Sigeman $\mathrm{H}$, et al. (2021) Avian neo-sex chromosomes reveal dynamics of recombination suppression and $w$ degeneration. Molecular Biology and Evolution.

64. Sun Y, Svedberg J, Hiltunen M, Corcoran P, Johannesson H (2017) Large-scale suppression of recombination predates genomic rearrangements in neurospora tetrasperma. Nat Commun $8(1): 1140$.

65. van Wyk S, Wingfield BD, De Vos L, van der Merwe NA, Steenkamp ET (2020) Genome-wide analyses of repeat-induced point mutations in the ascomycota. Front Microbiol 11:622368.

66. Kent TV, Uzunovic J, Wright SI (2017) Coevolution between transposable elements and recombination. Philos Trans $R$ Soc Lond B Biol Sci 372(1736).

67. Levy A, Schwartz S, Ast G (2010) Large-scale discovery of insertion hotspots and preferential integration sites of human transposed elements. Nucleic Acids Res 38(5):1515-30.

68. Paigen K, et al. (2008) The recombinational anatomy of a mouse chromosome. PLOS Genetics 4(7):e1000119.

69. Arbeithuber B, Betancourt AJ, Ebner T, Tiemann-Boege I (2015) Crossovers are associated with mutation and biased gene conversion at recombination hotspots. Proc Natl Acad Sci U $S$ A 112(7):2109-14

70. Halldorsson BV, et al. (2019) Characterizing mutagenic effects of recombination through a sequence-level genetic map. Science 363(6425).

71. Rousselle M, et al. (2020) Is adaptation limited by mutation? a timescale-dependent effect of genetic diversity on the adaptive substitution rate in animals. PLOS Genetics 16(4):e1008668.

72. Cioppi F, Casamonti E, Krausz C (2019) Age-Dependent De Novo Mutations During Spermatogenesis and Their Consequences. pp. 29-46.

73. Miyata T, Hayashida H, Kuma K, Mitsuyasu K, Yasunaga T (1987) Male-driven molecular evolution: a model and nucleotide sequence analysis. Cold Spring Harb Symp Quant Biol 52:863-7.

74. Rice WR (1984) Sex chromosomes and the evolution of sexual dimorphism. Evolution 38(4):735-742.

75. Hill WG, Robertson A (1966) The effect of linkage on limits to artificial selection. Genet Res 8(3):269-94.

76. Gurbich TA, Bachtrog D (2008) Gene content evolution on the $x$ chromosome. Current Opinion in Genetics Development 18(6):493-498.

77. Meiklejohn CD, Parsch J, Ranz JM, Hartl DL (2003) Rapid evolution of male-biased gene expression in $<\mathrm{em}>$ drosophila $<$ /em $>$. Proceedings of the National Academy of Sciences 100(17):9894-9899.

78. Parisi M, et al. (2003) Paucity of genes on the drosophila $x$ chromosome showing male-biased expression. Science (New York, N.Y.) 299(5607):697-700.

79. Lercher MJ, Urrutia AO, Hurst LD (2003) Evidence that the human $x$ chromosome is enriched for male-specific but not female-specific genes. Molecular Biology and Evolution 20(7):11131116.

80. Brekke TD, Henry LA, Good JM (2016) Genomic imprinting, disrupted placental expression, and speciation. Evolution 70(12):2690-2703.

81. Okamoto $\mathrm{I}$, Heard $\mathrm{E}$ (2006) The dynamics of imprinted $\mathrm{x}$ inactivation during preimplantation development in mice. Cytogenet Genome Res 113(1-4):318-24.

82. Scribner SJ, Wynne-Edwards KE (1994) Thermal constraints on maternal behavior during reproduction in dwarf hamsters (phodopus). Physiology Behavior 55(5):897-903.

83. Brekke TD, Good JM (2014) Parent-of-origin growth effects and the evolution of hybrid inviability in dwarf hamsters. Evolution 68(11):3134-3148.

84. Chapman JA, et al. (2011) Meraculous: De novo genome assembly with short paired-end reads. PLOS ONE 6(8):e23501.

85. Putnam NH, et al. (2016) Chromosome-scale shotgun assembly using an in vitro method for long-range linkage. Genome Research 26(3):342-350.

86. (2011).

87. McKenna A, et al. (2010) The genome analysis toolkit: A mapreduce framework for analyzing next-generation dna sequencing data. Genome Research 20(9):1297-1303.

88. Holt C, Yandell M (2011) Maker2: an annotation pipeline and genome-database management tool for second-generation genome projects. BMC Bioinformatics 12(1):491.

89. Langmead B, Salzberg SL (2012) Fast gapped-read alignment with bowtie 2. Nature Methods 9(4):357-9.
90. Trapnell C, et al. (2012) Differential gene and transcript expression analysis of rna-seq experiments with tophat and cufflinks. Nature Protocols 7(3):562-578.

91. Camacho C, et al. (2009) Blast+: architecture and applications. BMC Bioinformatics 10(1):421.

92. Bolger AM, Lohse M, Usadel B (2014) Trimmomatic: a flexible trimmer for illumina sequence data. Bioinformatics 30(15):2114-2120.

93. Li H, Durbin R (2009) Fast and accurate short read alignment with burrows-wheeler transform Bioinformatics 25(14):1754-1760.

94. Li H, et al. (2009) The sequence alignment/map format and samtools. Bioinformatics 25(16):2078-2079.

95. (2013-2015).

96. Chen K, et al. (2009) Breakdancer: an algorithm for high-resolution mapping of genomic structural variation. Nature Methods 6(9):677-681.

97. Li H (2018) Minimap2: pairwise alignment for nucleotide sequences. Bioinformatics 34(18):3094-3100.

98. Robinson JT, et al. (2011) Integrative genomics viewer. Nature biotechnology 29(1):24-26.

99. Durand NC, et al. (2016) Juicer provides a one-click system for analyzing loop-resolution hi-c experiments. Cell Syst 3(1):95-8.

100. Durand NC, et al. (2016) Juicebox provides a visualization system for hi-c contact maps with unlimited zoom. Cell Syst 3(1):99-101.

101. Sirén J, Välimäki N, Mäkinen V (2014) Indexing graphs for path queries with applications in genome research. IEEE/ACM Trans. Comput. Biol. Bioinformatics 11(2):375-388.

102. Liao Y, Smyth GK, Shi W (2014) featurecounts: an efficient general purpose program for assigning sequence reads to genomic features. Bioinformatics 30(7):923-30.

103. Sarver BA, et al. (2017) Phylogenomic insights into mouse evolution using a pseudoreference approach. Genome Biology and Evolution 9(3):726-739.

104. Ranwez V, Harispe S, Delsuc F, Douzery EJP (2011) Macse: Multiple alignment of coding sequences accounting for frameshifts and stop codons. PLOS ONE 6(9):e22594.

105. Minh BQ, et al. (2020) lq-tree 2: New models and efficient methods for phylogenetic inference in the genomic era. Molecular Biology and Evolution 37(5):1530-1534.

106. Mendes FK, Hahn MW (2016) Gene tree discordance causes apparent substitution rate variation. Systematic Biology 65(4):711-721.

107. Pond SLK, Frost SDW, Muse SV (2004) Hyphy: hypothesis testing using phylogenies. Bioinformatics 21(5):676-679.

108. RCore Team (2017) R: A language and environment for statistical computing. $r$ foundation for statistical computing, vienna, austria. 
Table S1. Assembly statistics for Dovetail genome

Short-read assembly

Total Length (Mbp)

L50 (scaffold count)

N50 (Mbp)

L90 (scaffold count)

N90 (Mbp)

Busco (Eukaryota)

Single copy

Duplicated

Fragmented

Missing

Total
2,113.27

7016

0.08

30,428

0.015

246

7

25

25

303
Final Assembly

$2,119.20$

5

165.747

14

30.611

263

7

9

24

303 
Table S2. Chromosome-level anchoring of genome using Phodopus genetic map

\begin{tabular}{cccccc} 
Chromosome & Mbp & cM & $\begin{array}{l}\text { Marker } \\
\text { count }\end{array}$ & All scaffolds & Scaffolds > 1Mbp \\
1 & 317.65 & 122.51 & 208 & 3 & 2 \\
2 & 288.91 & 120.25 & 174 & 4 & 1 \\
3 & 258.08 & 96.92 & 148 & 3 & 2 \\
4 & 155.67 & 97.42 & 137 & 2 & 1 \\
5 & 291.62 & 122.99 & 134 & 6 & 5 \\
6 & 129.1 & 107.65 & 135 & 4 & 1 \\
7 & 224.74 & 86.16 & 118 & 1 & 1 \\
8 & 125.09 & 67.23 & 74 & 2 & 1 \\
9 & 32.54 & 48.68 & 68 & 3 & 1 \\
10 & 64.22 & 52.51 & 52 & 2 & 2 \\
11 & 37.12 & 54.88 & 46 & 2 & 2 \\
12 & 24.28 & 54.05 & 47 & 2 & 1 \\
13 & 30.61 & 45.14 & 42 & 1 & 1 \\
$X$ & 119.12 & 42.79 & 42 & 2 & 0 \\
unplaced & 20.41 & $N A$ & $N A$ & 7719 & 1 \\
\hline
\end{tabular}


Table S3: Comparison of features on the $\mathrm{X}$ chromosomes of dwarf hamster and rat

\begin{tabular}{|c|c|c|c|c|c|c|c|c|}
\hline & $\begin{array}{l}\text { Length } \\
\text { (Mbp) }\end{array}$ & $\begin{array}{l}\text { Prop. } \\
\text { X }\end{array}$ & $\begin{array}{l}\text { gene } \\
\text { count, } \\
\text { gene } \\
\text { models }\end{array}$ & $\begin{array}{l}\text { gene } \\
\text { count, } \\
\text { with } \\
\text { pseudo } \\
\text {-genes }\end{array}$ & $\begin{array}{l}\text { Prop. } \\
\text { genes }\end{array}$ & $\begin{array}{l}\text { average } \\
\text { intergenic } \\
\text { distance, } \\
\text { gene } \\
\text { model } \\
\text { genes } \\
\text { only }\end{array}$ & $\begin{array}{l}\text { median } \\
\text { intergenic } \\
\text { distance, } \\
\text { gene } \\
\text { model } \\
\text { genes } \\
\text { only }\end{array}$ & $\begin{array}{l}\text { median } \\
\text { exon } \\
\text { length }\end{array}$ \\
\hline Phodopus X & 117.85 & & 697 & 896 & & 136402 & 41537.5 & 238 \\
\hline $\mathrm{Xq}$ & 41.22 & 0.350 & 318 & 385 & 0.544 & 105144 & 29915.5 & 236.5 \\
\hline $\mathrm{Xp}$ & 76.63 & 0.650 & 379 & 511 & 0.456 & 162698 & 56238.5 & 238 \\
\hline Rat X & 159.89 & & 824 & 1048 & & 156894 & 51211 & 282 \\
\hline $\mathrm{Xq}$ & 55.50 & 0.347 & 352 & 388 & 0.427 & 125683 & 40508 & 279 \\
\hline$X p$ & 104.39 & 0.653 & 472 & 660 & 0.573 & 180086 & 62730 & 291.5 \\
\hline
\end{tabular}


Table S4. Transposable element enrichment on X chromosome arms

Phodopus

$\begin{array}{ll}\text { Repeat } & \text { Repeat } \\ \text { genus } & \text { species }\end{array}$

recomb suppress . count . count

DNA

Academ

1

1

CMC-EnSpm 526

1270

$0.00261 X p$

DNA

64

149

1

Harbinge

8

12

hAT

5

5

hAT-Ac

123

185

0.4346

hAT-Charlie 731

1399

Mariner

1

Maverick

14

3

Tigger

228

353

0.0946

Tip99

27

27

0.29001

LINE

$$
\text { CR1 }
$$

LO

L2

LTR

$\begin{array}{llllrll}\text { ERV1 } & 642 & 1753 & 2.83 \mathrm{E}-11 \mathrm{Xp} & 1259 & 2659 & 0.01409 \text { Xp } \\ \text { ERVK } & 1842 & 3739 & 1 & 1358 & 3234 & 3.95 \mathrm{E}-12 \text { Xp } \\ \text { ERVL } & 777 & 1617 & 1 & 1904 & 3474 & 1\end{array}$

Rattus

enriched recomb suppress . count . count $P$ value arm

N/A N/A

$1668 \quad 2594$

4.30E-08 recomb.

$242 \quad 416 \quad 1$

$\begin{array}{lll}27 & 49 & 1\end{array}$

$\begin{array}{lll}14 & 9 & 0.22272\end{array}$

$\begin{array}{lll}442 & 829 & 1\end{array}$

$1029 \quad 1755 \quad 0.21794$

$\begin{array}{lll}3 & 5 & 1\end{array}$

$\begin{array}{lll}58 & 76 & 0.63574\end{array}$

$296 \quad 412 \quad 0.00181 \times q$

$\begin{array}{lll}26 & 29 & 0.7734\end{array}$

\#N/A \#N/A

$21923 \quad 38221 \quad 6.38 \mathrm{E}-15 \mathrm{Xq}$

$\begin{array}{lllll}0.00113 \times q & 36 & 20 & 0.00017 \times q\end{array}$

$41 \quad 30$

$0.00113 \times 9$

3474

nriched

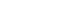

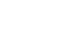




\begin{tabular}{|c|c|c|c|c|c|c|c|}
\hline & ERVL-MaLF & 3212 & 6783 & $0.04414 \mathrm{su}$ & 7720 & 13691 & 0.00068 recomb \\
\hline & Gypsy & 31 & 82 & 1 & 56 & 118 & 1 \\
\hline & Ngaro & 9 & 170 & $7.48 E-15 X p$ & 18 & 19 & 0.92928 \\
\hline $\begin{array}{l}\text { Retro- } \\
\text { transposon }\end{array}$ & & 287 & 575 & 1 & 459 & 706 & $0.01585 \mathrm{Xq}$ \\
\hline rRNA & rRNA & 15 & 28 & 1 & 10 & 38 & 0.62725 \\
\hline SINE & Alu & 10451 & 19278 & 5.02E-07 Xq & 18038 & 30379 & $6.38 \mathrm{E}-15 \mathrm{Xq}$ \\
\hline & B4 & 951 & 1603 & $0.00300 \times q$ & 2342 & 3490 & $6.38 \mathrm{E}-15 \mathrm{Xq}$ \\
\hline & ID & 331 & 666 & 1 & 2888 & 5162 & 0.4038 \\
\hline & MIR & 151 & 165 & 7.16E-06 Xq & 211 & 227 & $2.02 \mathrm{E}-07 \mathrm{Xq}$ \\
\hline & SB1 & 1577 & 2961 & 1 & 6155 & 9844 & $6.38 \mathrm{E}-15 \mathrm{Xq}$ \\
\hline snRNA & snRNA & 25 & 54 & 1 & 73 & 125 & 1 \\
\hline tRNA & tRNA & 2 & 0 & 1 & 7 & 7 & 1 \\
\hline Satellite & Satellite & 3 & 3 & 1 & 56 & 129 & 1 \\
\hline $\begin{array}{l}\text { Simple } \\
\text { repeat }\end{array}$ & $\begin{array}{l}\text { Simple } \\
\text { repeat }\end{array}$ & 28456 & 58384 & 6.06E-06 Xp & 22316 & 35728 & $6.38 \mathrm{E}-15 \mathrm{Xq}$ \\
\hline $\begin{array}{l}\text { Low } \\
\text { complexity }\end{array}$ & $\begin{array}{l}\text { Low } \\
\text { complexity }\end{array}$ & 5483 & 11451 & $0.01818 X p$ & 3136 & 5189 & $3.61 \mathrm{E}-07 \mathrm{Xq}$ \\
\hline Unknown & Unknown & 1305 & 3129 & $9.50 E-08 \times p$ & 992 & 1870 & 1 \\
\hline tRNA core & tRNA core & 101 & 122 & $0.00722 \times q$ & $\mathrm{~N} / \mathrm{A}$ & $\mathrm{N} / \mathrm{A}$ & \\
\hline
\end{tabular}


Table S5: Counts of tissue-enriched genes by chromosome, and BH corrected p-values Count

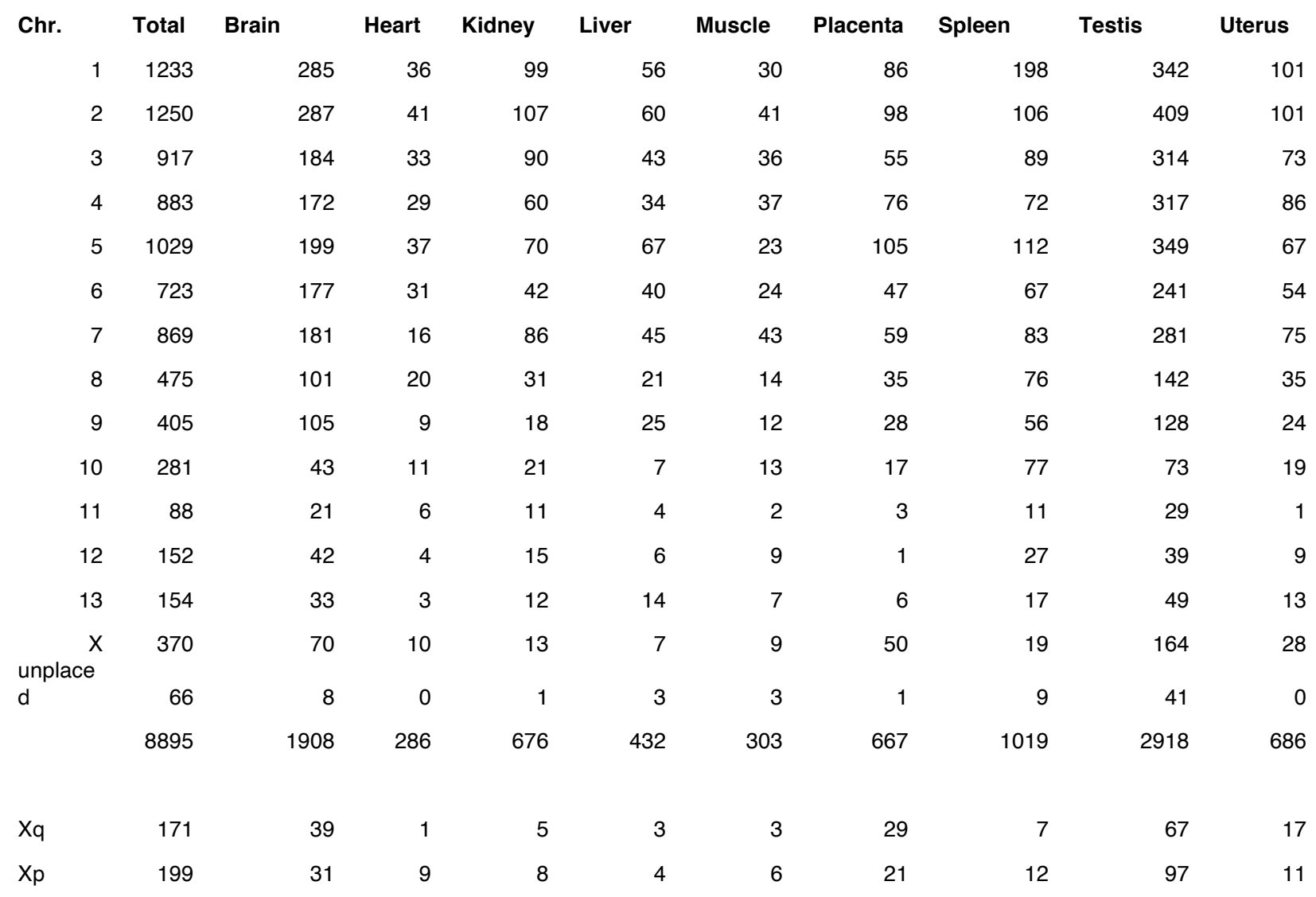

\section{Adjusted Pval}

\begin{tabular}{|c|c|c|c|c|c|c|c|c|c|}
\hline Chromosome & Brain & Heart & Kidney & Liver & Muscle & Placenta & $\begin{array}{l}\text { Spleen } \\
0.0011846\end{array}$ & Testis & Uterus \\
\hline 1 & 1 & 1 & 1 & 1 & 1 & 1 & 5 & 1 & 1 \\
\hline 2 & 1 & 1 & 1 & 1 & 1 & 1 & 1 & 1 & 1 \\
\hline 3 & 1 & 1 & 1 & 1 & 1 & 1 & 1 & 1 & 1 \\
\hline 4 & 1 & 1 & 1 & 1 & 1 & 1 & 1 & 1 & 1 \\
\hline 5 & 1 & 1 & 1 & 1 & 1 & 0.370845 & 1 & 1 & 1 \\
\hline 6 & 1 & 1 & 1 & 1 & 1 & 1 & 1 & 1 & 1 \\
\hline 7 & 1 & 1 & 1 & 1 & 1 & 1 & 1 & 1 & 1 \\
\hline 8 & $\begin{array}{r}1 \\
0.0005577\end{array}$ & 1 & 1 & $\begin{array}{r}1 \\
037091\end{array}$ & 1 & 1 & 0.315248 & 1 & 1 \\
\hline 9 & 2 & 1 & 1 & 2 & 1 & 1 & 0.0197259 & 0.97726 & 1 \\
\hline 10 & 1 & 1 & 1 & 1 & 1 & 1 & $2.49 \mathrm{E}-14$ & 1 & 1 \\
\hline 11 & 1 & 1 & 1 & 1 & 1 & 1 & 1 & 1 & 1 \\
\hline 12 & 0.0025056 & 1 & $\begin{array}{r}.40945 \\
6\end{array}$ & 1 & .20120 & 1 & 0.0027027 & 1 & 1 \\
\hline 13 & 1 & 1 & 1 & 1 & 1 & 1 & 1 & 1 & 1 \\
\hline$X$ & 1 & 1 & 1 & 1 & 1 & 1.44E-08 & 1 & 3.43E-15 & 1 \\
\hline
\end{tabular}


bioRxiv preprint doi: https://doi.org/10.1101/2021.11.15.468705; this version posted January 4, 2022. The copyright holder for this preprint (which was not certified by peer review) is the author/funder. All rights reserved. No reuse allowed without permission.

unplace

d

$\mathrm{Xq}$

$\mathrm{Xp}$

$\begin{array}{rr}1 & 1 \\ 0.230845 & 1 \\ 1 & 1\end{array}$

$1 \quad 1$
0.16624

1

1

4

4

$1 \quad 8.84 \mathrm{E}-07$

$1 \quad 0.056994$
1

$0.0060634 \quad 2.11 E-13$

1

1

$1 \quad 4.31 \mathrm{E}-11$
1
0.44209

2

1 


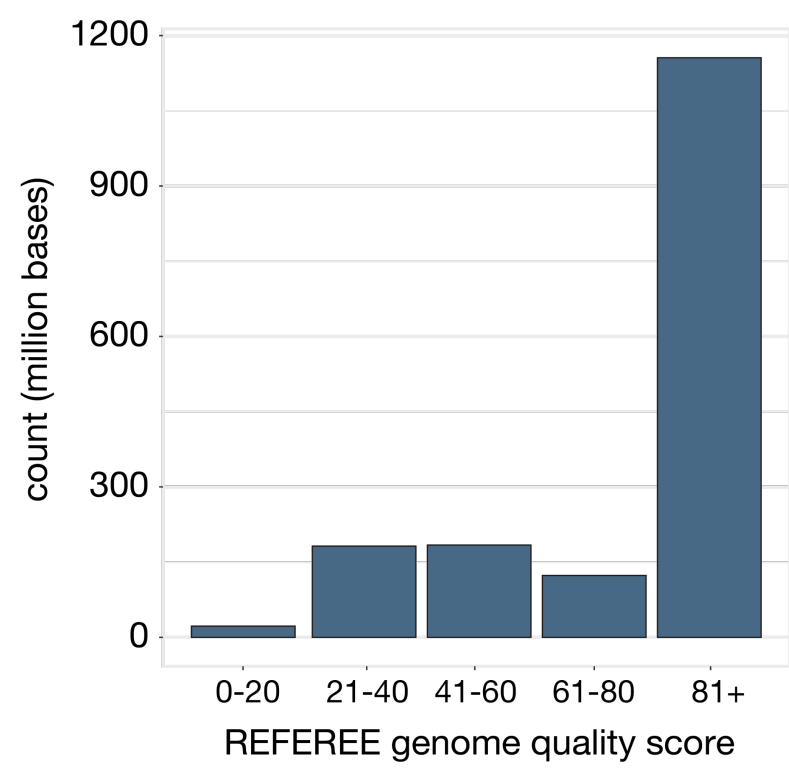

Supplementary Figure 1. Distribution of site-based quality scores from the largest scaffold per chromosome from Referee. 


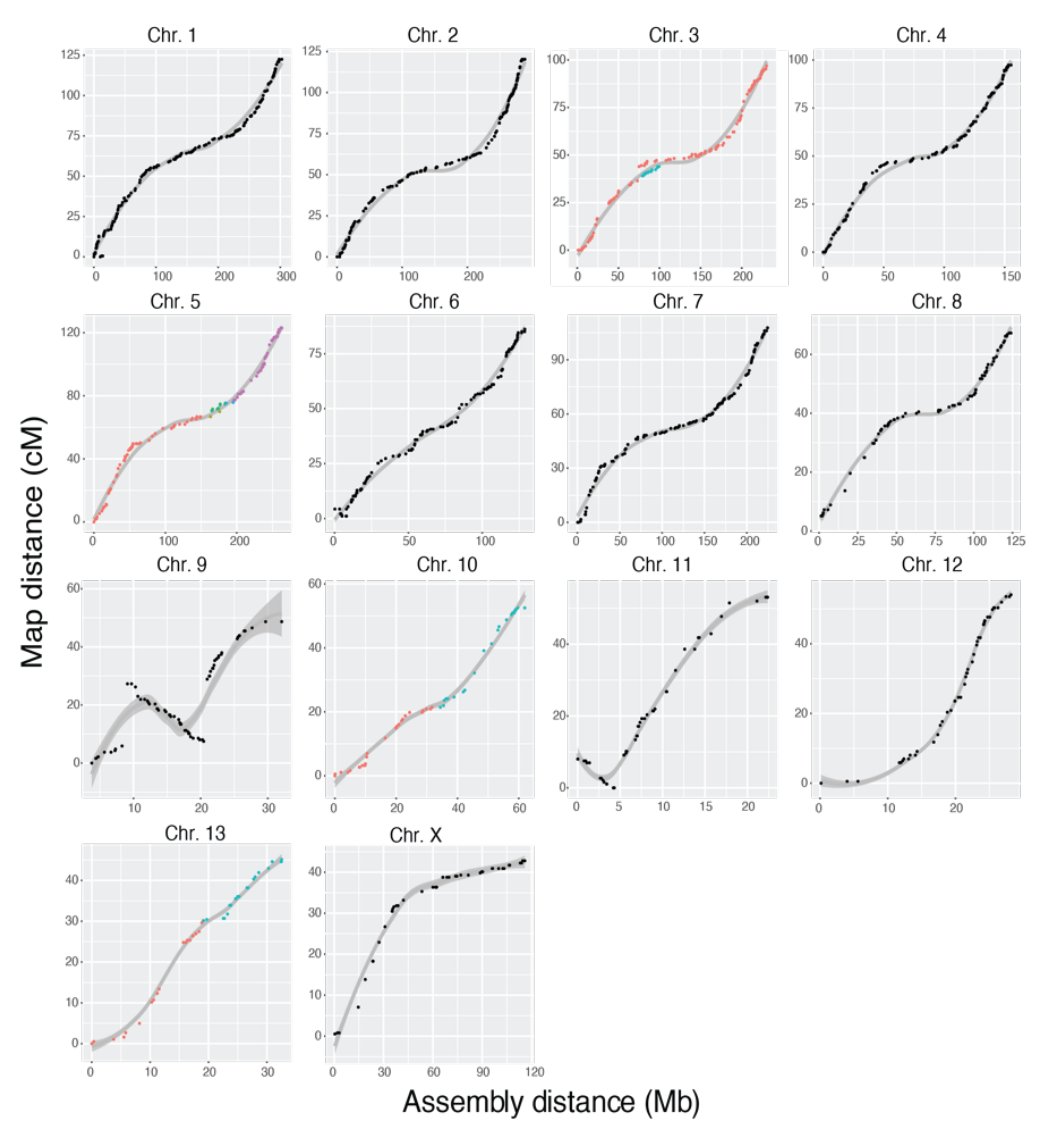

Figure S2. Marker positions by genetic map (cM) and assembly positions (Mb) for all chromosomes. Genetic and physical marker locations show recombination across each chromosome for all anchored scaffolds over $1 \mathrm{Mb}$, with the line showing a smoothed spline best fit. The slope of this line reflects recombination rate, with a steeper slope indicating a higher recombination rate. Metacentric chromosomes 1-8, 10, and 13 show an expected reduction in recombination rate near the centromere, whereas chromosome 12 shows an initial reduction in recombination that likely reflect acrocentric centromeres. Despite being metacentric, the $X$ chromosome never recovers recombination on the Xp arm. Regions of chromosome 9 and chromosome 13 with negative slopes likely reflect assembly errors. Colored points on chromosomes $3,5,10$, and 13 indicate scaffolds, all other chromosomes consist of one major scaffold. 

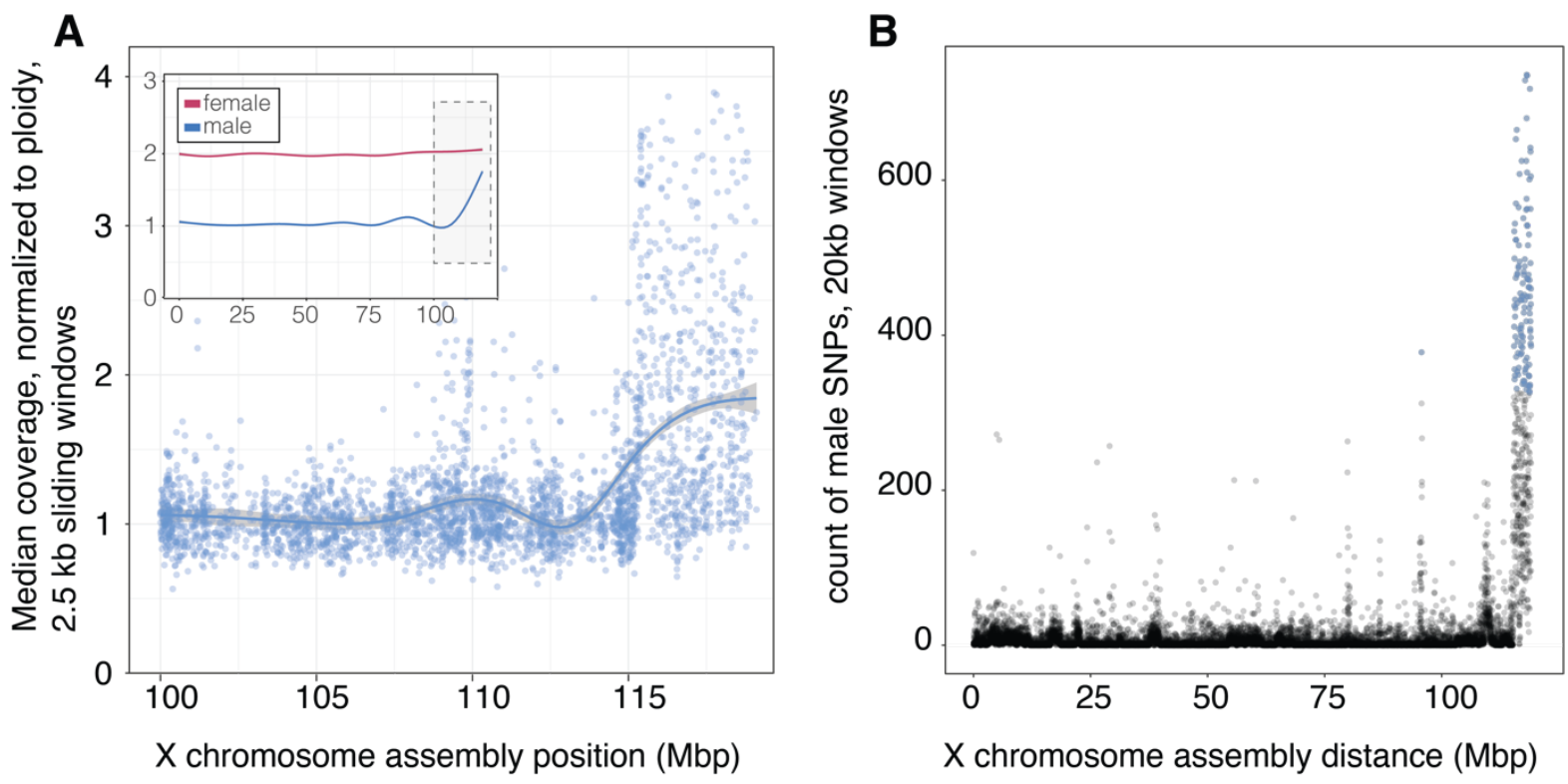

Supplementary Figure 3. Identification of the pseudoautosomal region of the $X$ chromosome. (A) Inset, median coverage normalized to ploidy along the entire $P$. sungorus $\mathrm{X}$ chromosome for males (blue) and females (female). The main panel shows the distal $X$ chromosome, where the pseudoautosomal region is indicated by an increase in coverage where reads from the $Y$ map to homologous sequence on the $X$. Mean coverage calculated in $2.5 \mathrm{kbp}$ sliding windows. (B) The distal end of the chromosome also shows an increase in the number of SNPs called from the male sequence, suggesting that these reads come from divergent $Y$ sequence. Count of SNPs in $20 \mathrm{kbp}$ windows. For (B), blue points indicate windows in the top and bottom $1 \%$ of the distribution of values. 


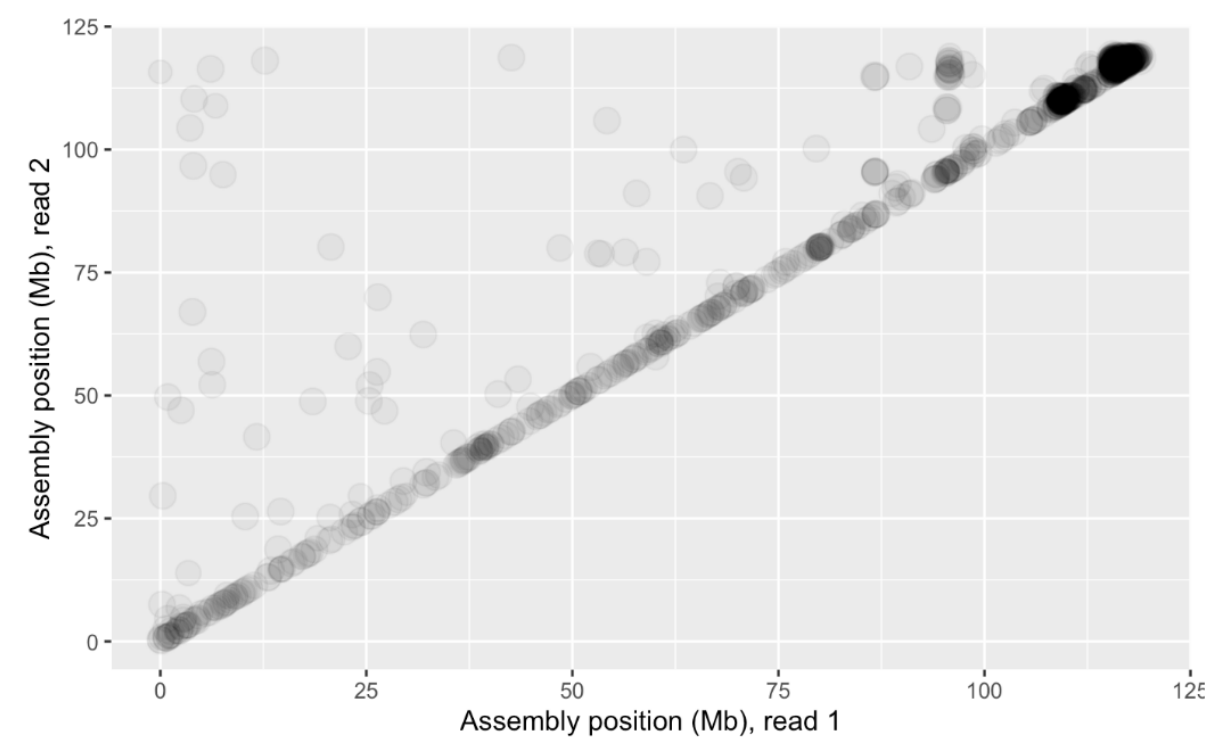

Supplemental Figure 4. Short-read support an inversion between $P$. sungorus and $\boldsymbol{P}$. campbelli on the $\mathrm{X}$ chromosome. Differences in the mapping location of read 1 (x-axis) and read 2 (y-axis) in a read-pair indicate an inversion in $P$. campbelli, using short-reads from $P$. sungorus as a baseline. Darker colors indicate more support for an inversion in this location. 

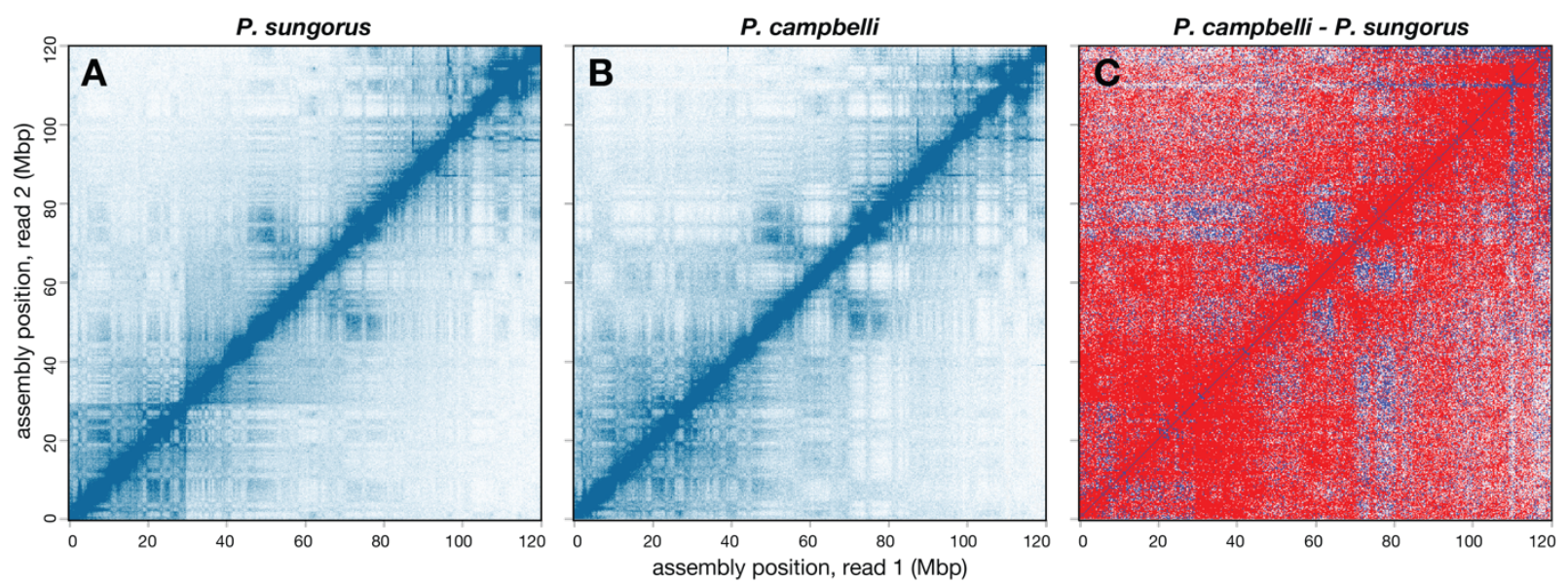

Supplemental Figure 5. Comparative chromatin configuration of the $X$ chromosome between $P$. sungorus and $P$. campbelli. HiC chromatin interactions, show short- and long-range interactions between points on the $\mathrm{X}$ chromosome, $250 \mathrm{Kbp}$ resolution, square root coverage normalization for (A) $P$. sungorus and (B) P. campbelli. (C) The difference between the two, where blue indicates increased contact in the $P$. sungorus chromatin map relative to the $P$. campbelli map. 

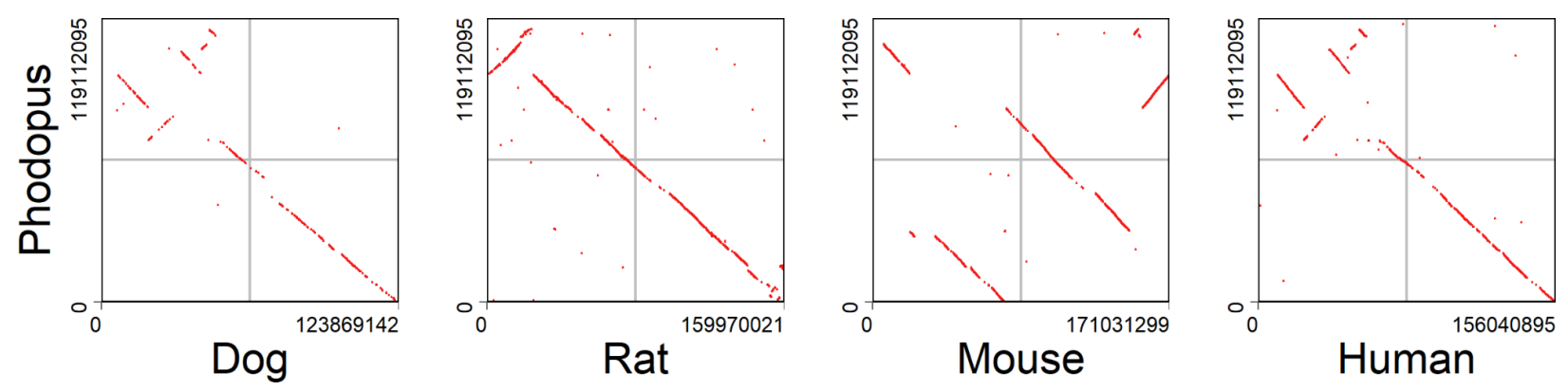

Supplemental Figure $6 . X$ chromosome synteny between mammalian species. Alignments of the $X$ chromosomes of dwarf hamster to domestic dog (CanFam3.1), rat (rnor6), mouse (mm10), and human (GRCh38) show broad conservation of synteny across mammals with the exception of mouse. 

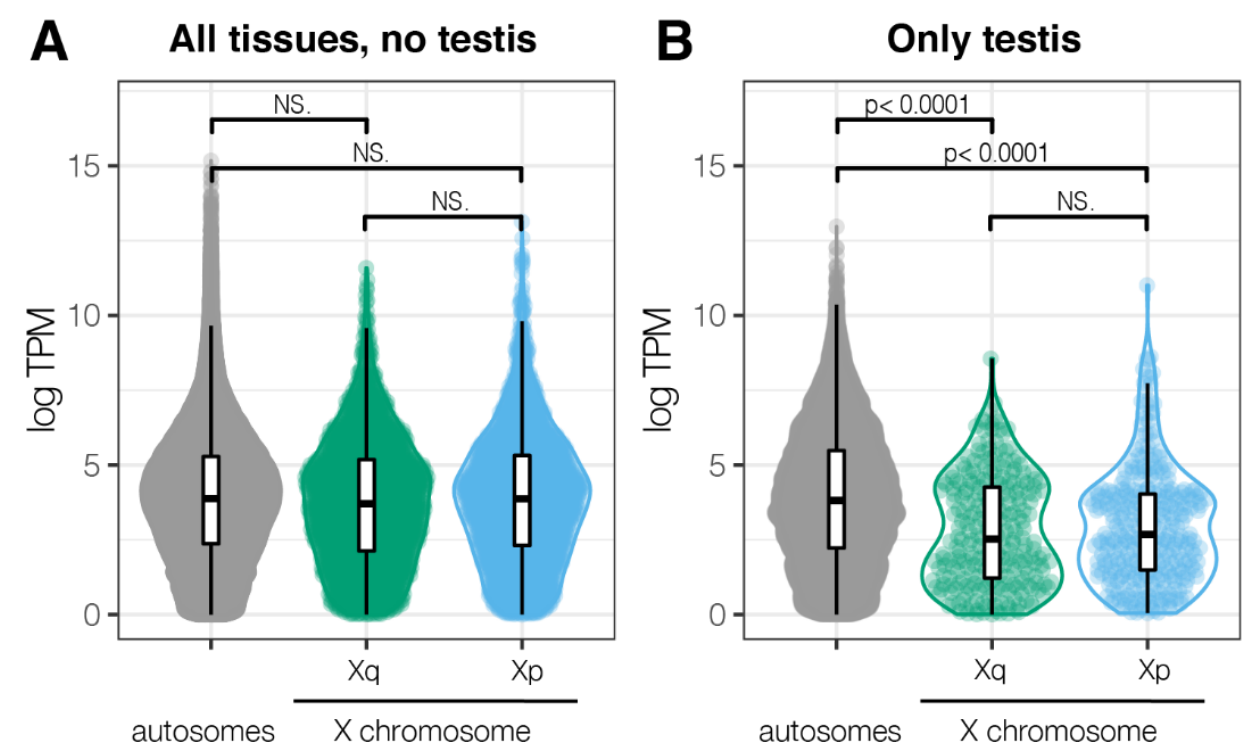

Supplemental Figure 7. Gene expression levels on the $X$ chromosome arms vs autosomes. Gene expression levels $\left(\log _{2}\right.$ TPM [transcripts per million] for (A) all tissues expect testis and $(B)$ testis (significance, pairwise Wilcoxon). 

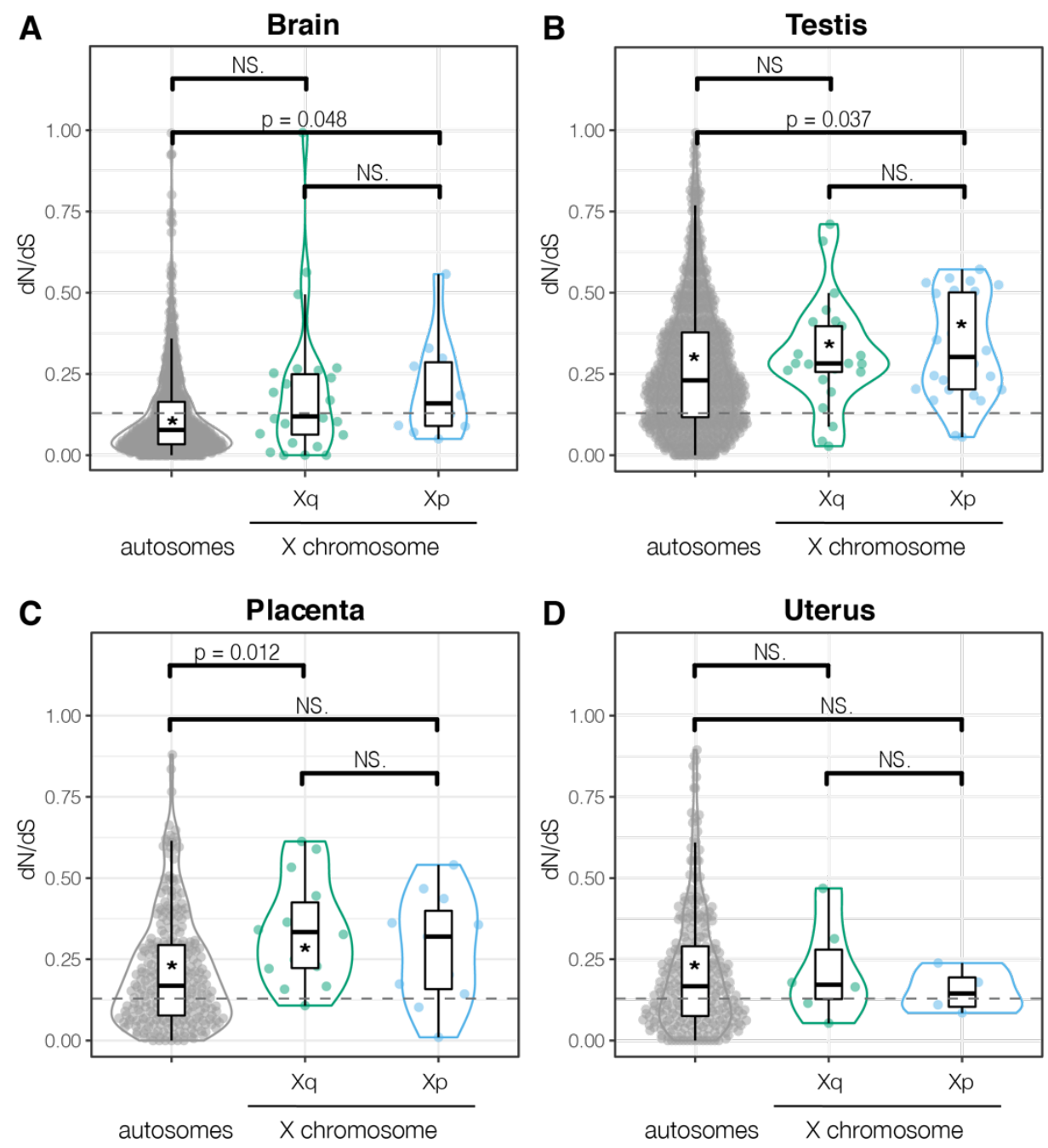

Supplemental Figure 8. Evolutionary rates in genes with high reproductive tissue specificity. $\mathrm{dN} / \mathrm{dS}$ for genes with Tau greater than 0.8 , for four tissues with sufficient numbers of genes on both $\mathrm{X}$ chromosome arms. Significance between autosomes and $X$ arms indicated with bars and corrected p-values (pairwise Wilcoxon). Dashed line indicates genome-wide $\mathrm{dN} / \mathrm{dS}$ for all genes, all tissues; significant deviation from the genome-wide median $\mathrm{dN} / \mathrm{dS}$ values is indicated with an asterisk $\left({ }^{*}\right)$ in the box plot (pairwise Wilcoxon). 


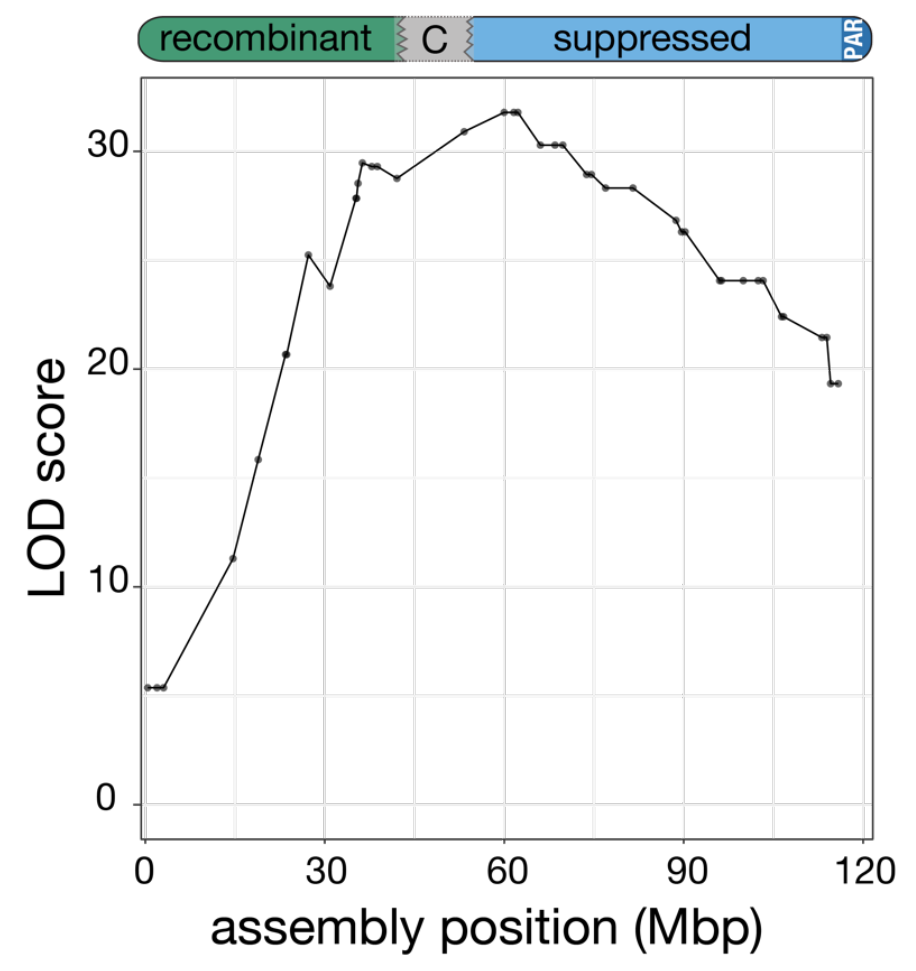

Supplemental Figure 9: Placental hybrid incompatibility QTL position on the $X$ chromosome genome build. Significance of association (LOD score) for X-linked hybrid incompatibility QTL shown according to marker assembly location (in Mbp), rather than genetic map (data from Brekke et al 2021) 

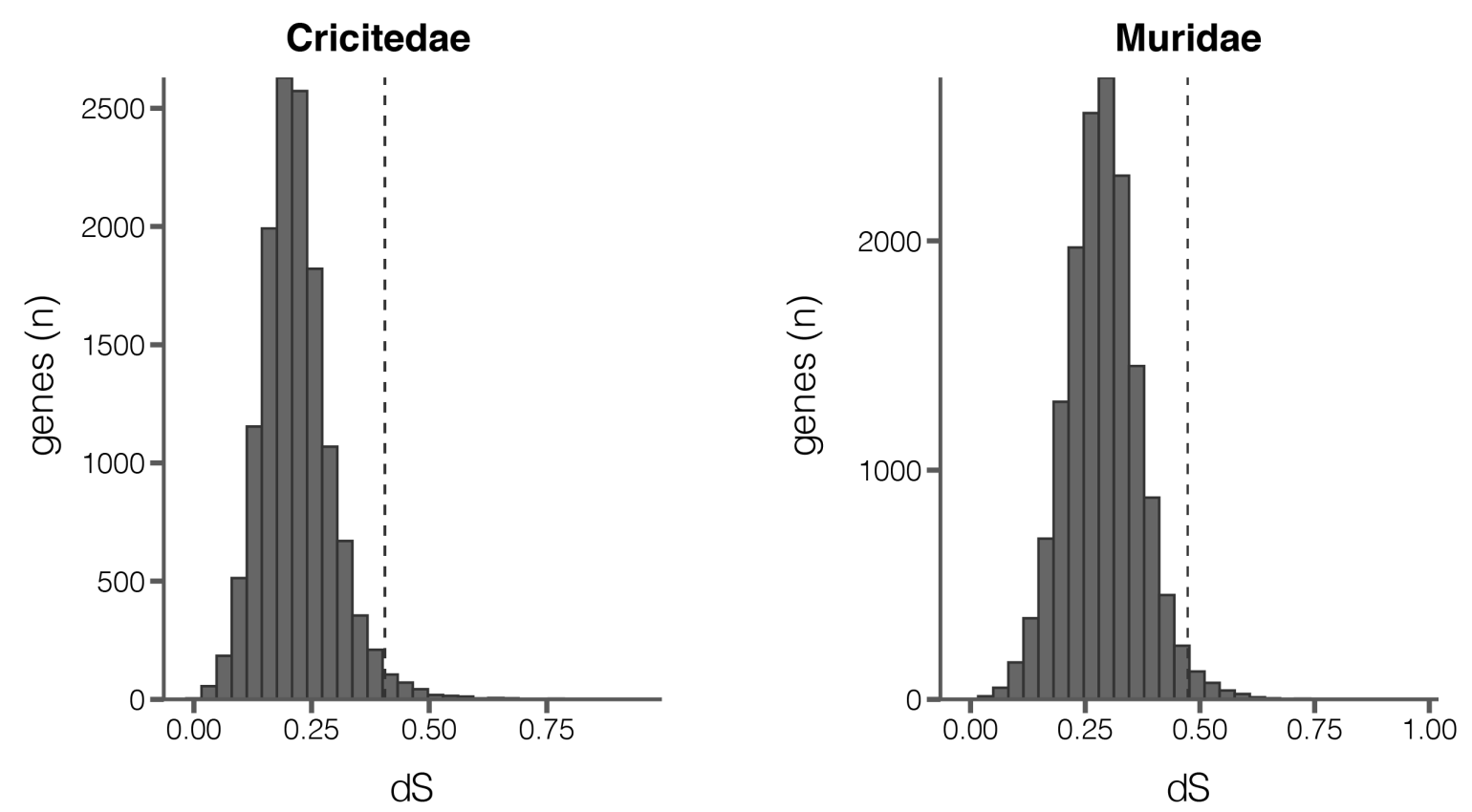

Supplemental Figure 10: Distributions of dS among single copy orthogroups identified in four species in Cricitedae and four species in Muridae. The vertical dashed line represents the $98^{\text {th }}$ percentile, above which genes were removed from subsequent analysis as a control for possible alignment error. 